\title{
Discovery and Use of Causal Patterns in Databases
}

\author{
David Bell, Francis McErlean ${ }^{\dagger}$ and Jiwen Guan \\ University of Ulster at Jordanstown, School of Information \\ and Software Engineering, Faculty of Informatics, \\ Newtownabbey, Co. Antrim Northern Ireland \\ e-mail:da.bell@ulst.ac.uk
}

\begin{abstract}
We consider here a variety of ways of using the attribute values of a database to obtain a single graphical model of cause and effect relationships, from the huge number of possible graphs available (quadratic in the number of attributes, at best). In our method a number of models are obtained from a variety of algorithms. In the paper we also outline our method of reasoning using a generalization of the Bayesian approach to the decomposition of complex problems. Matrices and their products are used to express the reasoning algorithms, making them simple and efficient to implement. These generation and manipulation techniques should be considered as part of an experimental system called Mining Kernel System (MKS) developed by the authors and colleagues, which links such techniques to a variety of data management systems, such as Ingres and Oracle. The primary objective of this paper is to identify the challenges of this novel research, which links knowledge and database engineering techniques, and its associated application pull, and to stimulate further research into the issues arising.
\end{abstract}

\section{KEYWORDS}

knowledge discovery in databases, data mining, automated reasoning, causality, evidential causal updating, decision trees

\footnotetext{
${ }^{\dagger}$ We are sad to report that Francis McElean died in November 1998.
} 


\section{INTRODUCTION}

This paper addresses the problems of understanding causation and of managing the distillation and use of causal networks which are ab initio latent in databases. These networks generalize rules, the usual objects of Knowledge Discovery in Databases (KDD), to include more of what would conventionally be considered domain knowledge. This area of database investigation is relatively new, and many practical and theoretical questions have been raised during the present study.

In the present context, this domain knowledge is distilled during the KDD process (that is, rather than being used in the database generation process) and it is represented in the graphical relationships between events, presences, happenings, and occurrences, the nodes of the graph, and the rules, similar to the edges of the graph. The graph or causal network captures the interrelations between rules in an easily accessible manner, and it can be used subsequently to update the graph in evidential reasoning activities.

The intention of this report is to

a) give an overview of the system; and

b) propose some algorithms for use in both parts of the system, giving some idea of their value.

It is important to verify clearly the applicability of the suggested techniques by systematically examining the purpose, objective, function, and scope of our Causal Network Management System (CANEMAS), and the first part of the paper does this.

In Sec. 2 of this paper we look at the motivation of this study and its perceived objective and purpose. This is illustrated by a motivating example which we have been using in the course of an application we are currently engaged in for the telecommunications industry. We investigate the scope of our techniques by outlining some basic concepts encountered when contemplating the management of causal relationships.

The heart of the paper is concerned with the function of the system. In Sec. 3, we show briefly how to distil the causal graph from a given database and how to use it subsequently. The details of the distillation algorithms are given in Sec. 4. They have all been implemented in our laboratory. A bread- 
board prototype based on this approach has already been implemented and is being applied in our telecommunication application.

In McErlean et al. (in press) we updated and propagated beliefs expressed in tree structures that were generated by one simple heuristic algorithm (HNG), using a simple database only. In this paper, because we use more general methods, we generate polytrees as well as trees using configuration algorithms such as $C A E G A$ and $O M T$. When we update the graphs, therefore, we also must be capable of updating polytrees. In Sec. 7 we outline a more general method of propagation than that presented in McErlean et al.

\section{THE PURPOSE, OBJECTIVE, AND SCOPE OF CANEMAS}

A major task in understanding states of affairs of the world as we see it is the accounting for and explanation of causal relationships among propositions (or variables or events or happenings, and so on). When there are many such relationships between dependent pairs of related propositions, we can usefully represent the whole picture by devices known as causal networks. Pearl (1986) has designed a scheme in which the latter are directed acyclic graphs in which the nodes represent propositions, the edges represent direct dependencies between interrelated variables, and the strengths of these dependencies are quantified by conditional dependencies or support parameters. The links in these networks are used not only for storing factual knowledge but also for directing and activating the data flow in the computations that manipulate this knowledge.

CANEMAS as a system is meant to support a particular kind of knowledge discovery in databases (KDD). KDD systems look for valuable patterns hidden in databases, and when they are discovered these are usually presented in the form of IF...THEN rules, usually having uncertainty measures attached. In CANEMAS, a structure of a particular (network) kind, governing the interworking of many rules, is sought. This additional structure is often thought of as supplementary domain knowledge. We have designed and implemented a prototype, Mining Kernel System (MKS) (Anand et al., 1996; Magill et al., 1995), within which individual rules may also be generated. The present work can be seen as an extension of MKS, but we present CANEMAS 
here as a stand-alone prototype. The prototype combines the discovery of the network with devices for its use in applications.

Several causal network generation algorithms have been used to supplement the machine-learning, clustering, and other numerical techniques in MKS, which have been described elsewhere in the literature (Agrawal et al., 1993; Anand et al., 1996). There are two nice things about MKS. One is that it provides a shared set of flexible components for building robust algorithms, in a manner that avoids duplicating work. The other attractive feature is that it provides flexible access to a variety of relational databases managed by different software packages, such as Ingres and Oracle, and even distributed databases.

As well as the qualitative, graphical side of our modeling approach, numbers are required so that probabilities can be assessed and propagated, and it is intended that MKS should support both aspects of our networks. An example of a source of the quantitative inputs required for network based reasoning is from the rule induction algorithms in MKS, whose outputs provide numbers such as uncertainty, support, and interestingness as a matter of course. These numbers can also be determined during the causal network generation phase of CANEMAS.

When rules have been discovered, they are combined with 'domain knowledge' to apply them to specific problems. For example, engineers might provide expert knowledge on how fault detection rules interact. The latter particular kind of domain knowledge is especially pertinent to the main telecommunications applications in our present study (see below). We have, however, experienced a 'user pull' recently to look at situations in which this background knowledge either is not available or is very sparse and fragmented. In these circumstances, it is desirable to discover the networked interactions between happenings, presences, events, and so on, using KDD tools.

The search for basic algorithms that we can use for this purpose has a long history, predating KDD as it is presently understood by a long time, as we indicate in Sec. 4. This rich heritage represents an excellent platform from which to launch our search for causal network distillation from databases, a function that is at the heart of CANEMAS.

Many activities rely on causal relationships in the solving of various problems and the making of various choices and decisions. A familiar problem is that where car mechanics may require to know the interrelations between faults and their manifestations to find what has to be done to repair a car. In 
the medical world, such detective work is also required, and other health related issues, such as dietary or environmental factors having a discerned probabilistic association with ill health, are also familiar. A causal network with information about the interaction between air pollution and asthma, and then that between asthma and worse respiratory diseases, could support the decision-making process. Once new data is supplied supporting any of these factors, the strength of the decision may be revised after carrying out an evidential reasoning process, which involves propagation of the new evidence throughout the complete network (see below).

Part of the motivation for network generation like this is that a generalpurpose kit bag of network extraction tools can be accumulated, which will be applicable to similar modeling problems from various unrelated domains. This reflects the more general "kernel" paradigm of MKS. For example, 'shapeless' databases (often just 'heaps' of coded observations) are available in plant ecology, genetics, and several medical specialties, but our primary motivation is from applications arising in industry. Large complex systems, such as chemical plants and aspects of electricity plant operation (particularly startup), are contenders as applications for the proposed tools.

The following relation (EVENTS) describing some features of the weather's effects on the heating-control systems of a house is presented as an illustrative and motivating example of the sort of input we provide to our network generation method (Table 1).

Clearly, the real Events database relation will be somewhat less directly amenable to manipulation, and some preprocessing will be needed to get it in this predominantly binary form.

Note that here only 9 of the $2^{5}(=32)$ possible binary combinations have been observed. Also notice that in a more general representation, ignorance could be represented in (non-relational) columns (Bell et al., 1996) by "attribute value" $\{0,1\}$.

There are five conventional database attributes here, and we have added a frequency attribute to indicate the number of tuples in the original database, characterized by each combination of events. The underpinning (simplified) causal network for this database (assumed unknown for our discovery activities, of course) might be conceived as representing the following relationships. 
TABLE 1

Database sample (EVENTS) storing 5 binary variables.

\begin{tabular}{|c|c|c|c|c|c|c|}
\hline & $\begin{array}{l}\text { Big Temp. } \\
U\end{array}$ & Warm & Warm & Therm. & Heavy & \\
\hline$u_{1}$ & 1 & 0 & 0 & 0 & 0 & 4 \\
$u_{2}$ & 0 & 1 & 0 & 0 & 0 & 6 \\
$u_{3}$ & 0 & 0 & 0 & 0 & 1 & 4 \\
$u_{4}$ & 1 & 0 & 0 & 1 & 0 & 4 \\
$u_{5}$ & 1 & 0 & 0 & 0 & 1 & 8 \\
$u_{6}$ & 1 & 1 & 0 & 1 & 0 & 6 \\
$u_{7}$ & 1 & 0 & 1 & 1 & 0 & 6 \\
$u_{8}$ & 1 & 1 & 1 & 1 & 0 & 14 \\
$u_{9}$ & 1 & 1 & 1 & 1 & 1 & 19 \\
\hline
\end{tabular}

A big temperature drop causes a thermostat flip in a house. The latter might then cause the corresponding room to warm up, and simultaneously ensure that there is warm water in the house. Part of a possible graph underpinning the set of observations in this relation is given in Fig. 1. The notation $M_{E \downarrow X}$ will be explained later. Notice that because this particular graph has not been completed, it would as it stands indicate that $x_{5}$ had no causal relationship with the other attributes (nodes), because the graph has no edge which includes $x_{5}$.

This simple example is used for clarity and, hopefully, intuitive appeal. The real applications envisaged are of much greater bulk and complexity. The specific system under consideration in our primary application is a network of multiplexers and other digital telecommunication components. We have been focusing on the modeling of one system component, a Synchronous Digital Hierarchy $(\mathrm{SDH})$ multiplexer, which transmits at rates of $155 \mathrm{Mbits} / \mathrm{sec}, 622$ $\mathrm{Mbits} / \mathrm{sec}$ and $2.5 \mathrm{Gbits} / \mathrm{sec}$, with the frame transmission rate of $8 \mathrm{KHz}$. The ultimate goal is to model a communication network of such components and to address the problem of enhancing the performance of fault handling. There are large numbers of causes (faults) and each of these can trigger other faults which are different combinations of "external" effects (signals). Causes can 


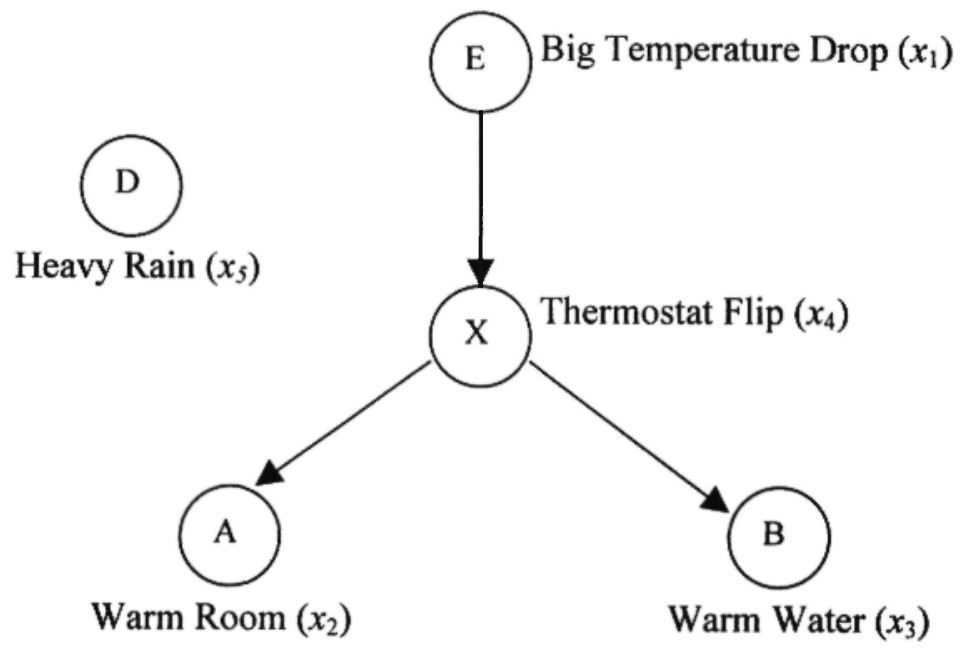

Fig. 1: An example of a causal network

have many effects and vice versa. Relationships between causes and effects tend to display a multiple level structure (vertically) and some such relationships are non-linear in nature for reasons not understood by the communication engineers. Cause and effect relationships can also be subject to statistical and probabilistic effects and to time delays.

To model this system, a causal network of well over 100 nodes is envisaged and this sort of problem is typical of sizeable family of such problems. In these problems, conventional engineering mathematics or other known relationships are of only limited utility because the cascading behaviour due to the multiple sequential and parallel internodal relationships is very complex and is poorly understood by the domain experts. Putting lots of microsolutions together to try to model macrobehavior is a daunting task and produces a rat's nest of horrific complexity. 
Up to now we have been restricting our attention to trees. A causal network, however, may result in the form of either a causal tree or a causal polytree (see Fig. 2a and $2 \mathrm{~b}$, respectively). In causal trees each node, apart from a root node such as node 5 in Fig. $2 \mathrm{a}$, has only one parent.

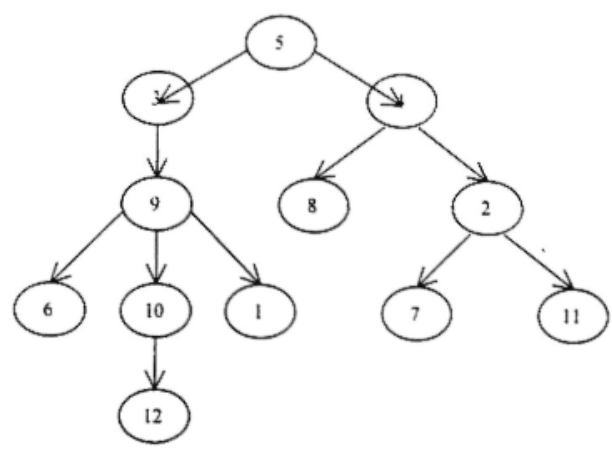

(a)

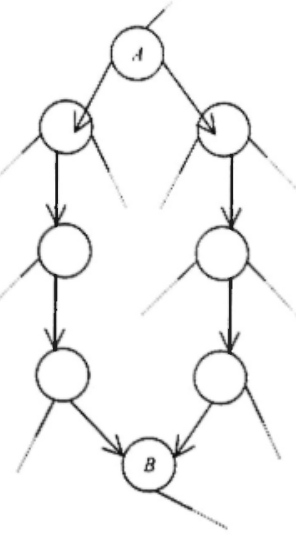

(c)

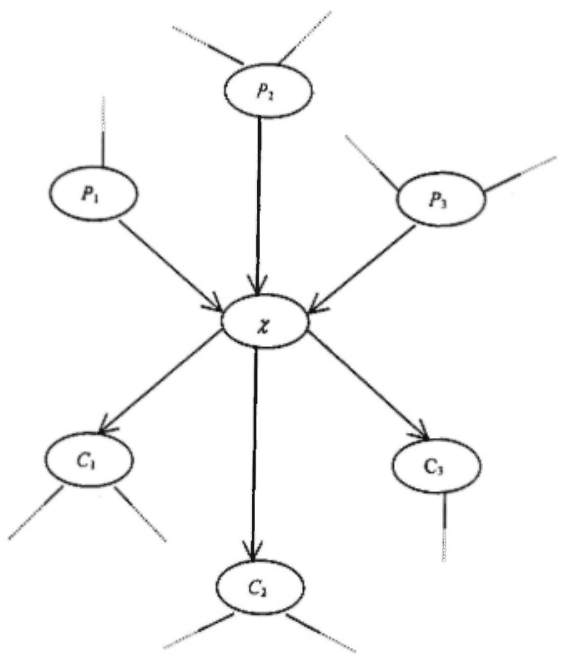

(b)

Fig. 2: (a) An example of a causal tree; (b) the parents and children of a node $\chi$ in a fragment of a polytree; and (c) a typical loop between nodes $A$ and $B$. 
On the other hand, a causal polytree can be viewed as a collection of several causal trees fused together at certain nodes, in such a way that more than one parent is permitted (see Fig. 2b). Now, in the general situation with such fused networks, loops can be present in the network, as illustrated in Fig. 2c. Causal polytrees do not contain such loops, and this permits new evidence to be used in an updating scheme similar to that used for causal trees.

Pearl (1988) has studied the updating of polytrees extensively. Section 4 discusses the different types of evidential causal networks and their features in further detail. Now it is well known that evidence theory (Shafer, 1976) is a generalization of classical Bayesian statistics, which suggests a generalization of Bayesian causal networks. Our hypothesis is that the advantages of evidence theory over Bayesian methods for some applications will carry over to networks. These applications include the Dempster-Shafer model developed by Gordon and Shortliffe (1984) for the purpose of handling uncertainty (in trees only) in the MYCIN system, which is used for medical reasoning. This evidential model is based on a firm mathematical foundation that has a clear advantage over MYCIN's more ad hoc certainty factors.

The second part of this paper deals with the use of these networks in evidential reasoning. The central concept in this part is belief propagation. We have developed this concept by investigating evidential causal networks, in which the strengths of the dependencies between interrelated nodes are represented as rule strength matrices. Matrices and their products are used to express the reasoning algorithms. In Sec. 7 we describe our reasoning algorithm (PropBel) for causal polytrees and discuss how it operates. In the sections before that, we indicate how causal networks are generated in our system and give an outline of the Dempster-Shafer Theory of Evidence. Section 8 presents some of the results we obtained using PropBel in our application in the telecommunications industry.

So, to summarize this section, the ultimate purpose of CANEMAS is to provide a computational model supporting reasoning under uncertainty which captures the concept of causation and can be used to extract causal relationships from evidence (held at multiple sources) and to support decision making, based on these relationships.

The objective is to accomplish this goal efficiently and in a manner that provides effective outputs, outputs that can clearly be demonstrated to be 
useful, to (engineering) professionals in our main application. This objective still represents a future goal. First indications of the value of the system in the application domain, its effectiveness, have been promising (Sterritt et al., 1997).

When extracting 'world models' from databases, we found the need to start out from a position in which we have a clear notion of what quality of our "Universe of Discourse" it is that we are trying to capture. This approach is particularly important in the case of causality. Rather than embark on a discussion of causality concepts which is, we confess, perhaps a little philosophical for the present forum, we ask the interested reader to consult Appendix B. We believe that some idea our concept of causality is essential to an understanding of the scope of our contributions. We have to be sure that the notion is clearly conceived. We have had to face up to the fact that such an understanding is essential if we are to assess our success (e.g. by testing) in the present study.

With our understanding of the concept of one event causing another analogy to relatively unambiguous canonical metaphors, we can proceed to build an apparatus which will be of some utility in handling many analogous cause and effect relationships in applications. In this way, the underpinning canonical cause and effect relationship will have enabled us, we hope, to incorporate the required semantic concept.

So the scope of our system is for causal relationships which are analogous, in the way stated above, to the canonical scenario in Appendix B. Our 'ideal physical world', analogous to (for example) the lines, points, and planes of Euclidean geometry, is one where, by ceteris paribus, we insist on for example a vacuum, fixed friction, fixed initial speeds, fixed temperature, and so on for the lifetime of our observations/experiments.

\section{THE FUNCTION OF CANEMAS}

As stated above, the function of CANEMAS has two components: (1) the distillation of the causal networks from the databases; and (2) the use of the network for applications.

The function is the key subject of the present paper. It has two main components, as we describe here, and we give more detail of the components 
in later sections. In this section we outline both aspects of the function of CANEMAS.

(a) An emerging possibility, due to the capabilities resulting from research and exemplar applications involving belief networks, is that bulky, complex application systems that defy full understanding using conventional domainrelated means can be modeled with some success as cause and effect networks, or causal networks.

A primary output of CANEMAS is graphic structure underpinning an ontology as represented as a relational database. This graphic model, the causal network, will be used to predict the physical system's behaviour as discussed in (b) below.

We outline here our approach to one of two aspects of structure generation, the qualitative aspect, using inputs that are available from such sources as distributed databases, other KDD tools, evidential reasoning techniques, and statistical databases. This logical structure distilled from a database of events will incidentally provide fallout, which will be very useful for those who wish to use the application system. For example, three-way links between faults (nodes) indicated as edges on sub-graphs, can help engineers form new hypotheses on the causal behaviour of complex systems. To use the system in practice, the quantitative elements of the model must also be derived. This procedure is relatively well understood and is not discussed further in this paper.

Many researchers in the causal network area claim that the generation of the networks should be done by humans. Despite this view, several automated approaches to this task have already been suggested. Examples from the literature include (a) Druzdel and Simon's (1993) structural equation approach using simple simultaneous equations; and (b) Cooper's numerical (probabilistic) method (Cooper \& Herskovits, 1992). We have implemented these approaches, and also our own methods based on rough sets (Pawlak, 1991), a method based on mutual information (Chow \& Liu, 1968), and a genetic algorithm based on method (Rojas-Guzman \& Kramer, 1993). For our telecommunication case study, we observed that each of these methods generates a different result! The outputs are presented in Sec. 4 . We are currently looking at methods of combining the output from these algorithms by using the genetic algorithm method or by using evidence theory itself. This model averaging needs careful study and is still an open research issue. 
Nevertheless, methods of scoring the models to be averaged have been suggested, e.g. by Cooper and Herskovits (1992).

Our approach is characterized as one that uses the evidence from many sources (in our case algorithm outputs) to help us choose the best graph. Evidence from subsidiary sources that are not so easily quantified, such as from domain knowledge inputs and from the direction of time flow, can also help eliminate some contenders for the networks.

(b) When using the network for reasoning about the properties of entities, we are often trying to either diagnose (argue from effect to causes) or to classify (argue from causes to effects). The method we use is based on evidence theory (Guan \& Bell, 1991; Shafer \& Srivastava, 1990); our method is therefore a generalization of Bayesian causal network methods. As indicated earlier, the use of our method to date has been confined to causal networks in which each node has only one incoming edge, in which each link has an associated causal matrix $M$ as defined below, and where there are no cycles. This scope can be extended considerably by the use of heuristic methods in much the same way as is done for Bayesian causal networks.

A typical node, say $X$, with corresponding values $\left\{X_{1}, \ldots, X_{w}\right\}$, represents a Boolean algebra

$$
<\chi, \cup, \cap,{ }^{\prime}, \varnothing>
$$

where

$$
\chi=\left\{\varnothing, X_{1}, X_{2}, \ldots, X_{b} \ldots, X_{w}\right\} ; n=|\chi|-1 ; X_{w}=\Psi ;
$$

and $U, \cap,{ }^{\prime}$ are union, intersection, and negation, respectively; $\varnothing$ is the zero element, the smallest in $\chi$ under the partial ordering relation $\subseteq$ determined by $U, \cap ; \Psi$ is the identify element, the biggest in $\chi$ under the partial ordering relation $\subseteq$ determined by $U, \cap$.

If $X$, is the answer to a theoretical question, then

$$
X_{1}, X_{2}, \ldots, X_{w}
$$

are hypotheses; if $\mathrm{X}$ is observable, then

$$
X_{1}, X_{2}, \ldots, X_{w}
$$

are observations. 
An edge from node $E$ to $X$ means that $E$ directly causes $X$. Let

$$
E=\left\{\varnothing, E_{1}, E_{2} \ldots, E_{j}, \ldots, E_{1}\right\} ; 1=|E|-1, \bar{c}_{1}=\Psi .
$$

The strength of this link (Guan \& Bell, 1991) is quantified by $l$ mass functions $\left(p_{j}(X), \forall j=1, \ldots, l\right)$, which are defined in Sec. 6 .

$$
p_{1}(X)=p_{1}\left(X \mid E_{1}\right), p_{2}(X)=p_{2}\left(X \mid E_{2}\right), \ldots, p_{j}(X)=p_{j}\left(X \mid E_{j}\right), \ldots, p_{l}(X)=p_{1}(X \mid \Psi)
$$

\begin{tabular}{|c|c|c|c|c|c|c|}
\hline$E \rightarrow X$ & $\mid x_{1}$ & $\ldots$ & $X_{i}$ & $\ldots$ & $X_{w}$ & $\sum_{i=1, \ldots, n} p_{j}\left(X_{i} \mid E_{j}\right)$ \\
\hline$E_{1}$ & $\mid p_{1}\left(X_{l} \mid E_{l}\right)$ & $\ldots$ & $\mathrm{p}_{1}\left(X_{i} \mid E_{1}\right)$ & $\ldots$ & $p_{1}\left(X_{w} \mid E_{1}\right)$ & 1 \\
\hline$E_{2}$ & $\mid p_{2}\left(X_{1} \mid E_{2}\right)$ & $\cdots$ & $\mathrm{p}_{2}\left(X_{1} \mid E_{2}\right)$ & $\cdots$ & $p_{2}\left(X_{w} \mid E_{2}\right)$ & 1 \\
\hline$\ldots$ & & $\ldots$ & $\cdots$ & $\ldots$ & $\ldots$ & 1 \\
\hline$E_{j}$ & $\mid p_{J}\left(X_{I} \mid E_{J}\right)$ & $\ldots$ & $p_{j}\left(X_{i} \mid E_{j}\right)$ & $\ldots$ & $p_{\jmath}\left(X_{w} \mid E_{\jmath}\right)$ & 1 \\
\hline$\ldots$ & $1 \ldots$ & $\ldots$ & $\ldots$ & $\ldots$ & $\ldots$ & 1 \\
\hline$E_{l}$ & $\mid p_{l}\left(X_{l} \mid E_{l}\right)$ & & $p_{l}\left(X_{l} \mid E_{l}\right)$ & & $p_{l}\left(X_{w} \mid E_{l}\right)$ & 1 \\
\hline
\end{tabular}

on $\chi$ as follows:

In fact, the link represented by the edge is a rule as follows.

$\operatorname{RULE}(E \downarrow X)$ :

IF EVIDENCE $E_{1}$ THEN

HYPOTHESIS $X_{1}$ WITH STRENGTH $p_{l}\left(X_{1} \mid E_{1}\right)$

...

HYPOTHESIS $X_{n}$ WITH STRENGTH $p_{l}\left(X_{n} \mid E_{1}\right)$

\section{......}

\section{ELSE IF EVIDENCE $E_{1}$ THEN}

HYPOTHESIS $X_{1}$ WITH STRENGTH $p_{l}\left(X_{1} \mid E_{l}\right)$

...

HYPOTHESIS $X_{n}$ WITH STRENGTH $p_{l}\left(X_{n} \mid E_{l}\right)$

This rule RULE $(E \downarrow X)$ corresponds to a causal matrix or rule strength matrix, which we denote $M_{\mathrm{E}} \downarrow_{\mathrm{X}}$, (see Fig. 1 above) with $l$ rows and $n$ columns

$$
M_{\mathrm{E} \downarrow X}=\left[\begin{array}{lllll}
p_{1}\left(X_{1} \mid E_{1}\right) p_{1}\left(\ddot{A}_{2} \mid \bar{E}_{1} ;\right. & \cdots & p_{1}\left(\ddot{A}_{i} \mid \bar{E}_{1} ;\right. & \cdots & p_{1}\left(X_{w} \mid E_{1}\right) \\
p_{1}\left(X_{1} \mid E_{2}\right) p_{1}\left(X_{2} \mid E_{2}\right) & \cdots & p_{1}\left(X_{i} \mid E_{2}\right) & \cdots & p_{1}\left(X_{w} \mid E_{2}\right) \\
\cdots & \cdots & & \cdots & \cdots \\
p_{1}\left(X_{1} \mid E_{j}\right) p_{1}\left(X_{2} \mid E_{j}\right) & \cdots & p_{1}\left(X_{1} \mid E_{j}\right) & \cdots & p_{1}\left(X_{w} \mid E_{j}\right) \\
\cdots & \cdots & & \cdots & \cdots \\
p_{1}\left(X_{1} \mid E_{l}\right) p_{1}\left(X_{2} \mid E_{l}\right) & \cdots & p_{1}\left(X_{1} \mid E_{l}\right) & \cdots & p_{1}\left(X_{w} \mid E_{l}\right)
\end{array}\right]
$$


In the case of the graph in Fig. 1, using the values in Table 1 we obtain the following matrix for our running example.

\begin{tabular}{l|lll}
$M_{x 1} \downarrow_{x 4}$ & $\{0\}$ & $\{1\}$ & $\{0,1\}$ \\
\hline$\{0\}$ & $10 / 10=1$ & $0 / 10=0$ & $0 / 10=0$ \\
$\{1\}$ & $12 / 61=0.197$ & $49 / 61=0.803$ & $0 / 61=0$ \\
$\{0,1\}$ & 0 & 0 & 1
\end{tabular}

Notice that we included the third row $[0,0,1]$, corresponding to $\{0,1\}$ to represent the ignorance inherent in using the limited representation available in a database. This indicates the major advantage of using evidential rather than Bayesian methods. The evidential alternative to the Bayesian causal network is attractive for the database application because the closed world of the database is in fact a non-full representation of the real world ontology, and the explicit accounting for ignorance in evidence theory is therefore a considerable advantage. Ignorance in the probability world means reserving a part of one's belief, e.g., not allocating it to any of the contending decisions. In the database world we would also like this capability, because part of the 'world' represented by the database is more extensive than the database contents (e.g., not possible that all tuples are present in the database).

In causal networks, every node can be activated for reasoning purposes as soon as the activating data become available. The reasoning process includes three steps which are simply listed here:

Step 1. activating data;

Step 2. updating belief;

Step 3. propagating belief.

When equilibrium is reached, each node (event) $X$ will be assigned a mass function $\mathrm{m}(X)$ (Bell et al., 1996). So matrices and their products are used to express the reasoning algorithms one of which is discussed in Sec. 7.

\section{ALGORITHMS AND EXPERIMENTS IN CAUSAL NETWORK DISTILLATION}

As stated earlier there is a fine legacy dating back to the 1950 s of algorithms that can be considered as contenders for the generation of causal 
networks from databases. We outline some of these below and supplement them with some algorithms of our own. They are available in CANEMAS for use as individual graph generators or (as described here) for finding a consensus from a number of such devices.

\subsection{Simon's Algorithm}

Models, which are simplifications of the reality, can be represented by sets of simultaneous equations, in which each equation describes a functional relation among a subset of the model's variables. Variables exogenous to a subset are the direct causal predecessors of the variables in that subset. The equations often contain so-called error variables that are exogenous and usually independent by assumption, and that represent the joint effect of other variables that cannot be specified. A generic form of an equation is

$$
f\left(x_{1}, x_{2}, \ldots, x_{n}, \varepsilon\right)=0
$$

where $f$ is an algebraic function, its arguments $x_{1}, \ldots, x_{n}$ are system variables, and $\varepsilon$ is an error variable. The diagram below shows the corresponding structural equations for a sample binary database with four attributes $(w, x, y, z)$.

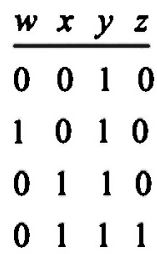

$$
\left\{\begin{aligned}
y & =\varepsilon_{1} \\
w+C_{1 y} & =\varepsilon_{2} \\
x+C_{2 y} & =\varepsilon_{3} \\
x+C_{3 y}+C_{4 z} & =\varepsilon_{4}
\end{aligned}\right.
$$

$C_{1}, \ldots, C_{4}$ are non-zero constants, and the error variables $\varepsilon_{1}, \ldots, \varepsilon_{4}$ are specified by a probability distribution. A change in $\varepsilon_{1}$ will affect the value of $y$ directly, and the values of $w, x$, and $z$ indirectly. A change in $\varepsilon_{4}$, however, will only affect $x$. The set of structural equations is self-contained (i.e., they consist of as many equations as variables). Each subset of $m$ equations is self-contained too and is known to be of order $m$. Simon's procedure identifies minimal self-contained subsets (i.e., those that do not have self-contained subsets), solves them for the variables they contain, and substitutes the values in the remaining equations. 
The resulting cause and effect network structure for the binary database above is

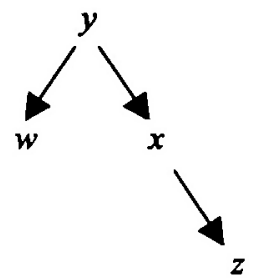

Variable $y$ is the exogenous variable of this structure ; the remaining ones are endogenous. The pseudocode of Simon's approach (Druzdzel \& Simon, 1993) is shown below.

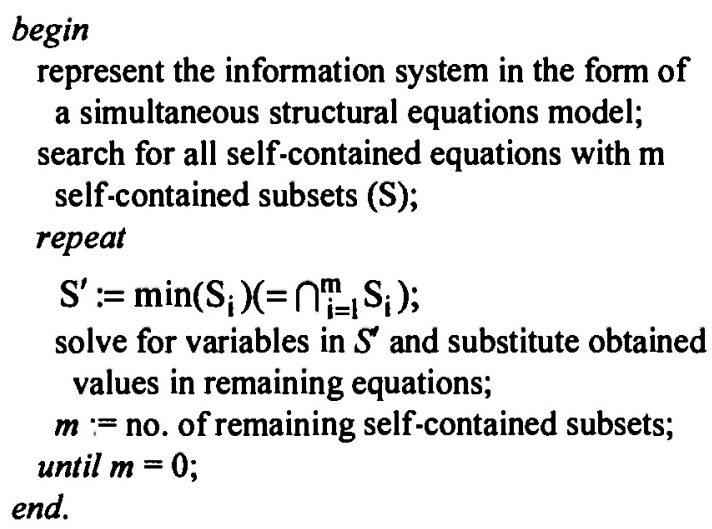

\subsection{The OMI Algorithm}

The optimization of mutual information $(O M I)$ method measures, information theoretically, the closeness between each pair of variables. It is based on the assumption that the uncertainty regarding any variable $X$ characterized by a probability distribution $P(X)$ can be represented by the Shannon entropy function (Shannon \& Weaver, 1949)

$$
H(X)=-\sum_{x} P(x) \log P(x) .
$$

The joint entropy is given by

$$
H(X)=-\sum_{x, y} P(x, y) \log P(x, y)
$$

and the conditional entropy is 


$$
H(X \mid Y)=-\sum_{x, y} P(x, y) \log P(x \mid y)
$$

These two entropies are related by

$$
\begin{aligned}
H(X, Y) & =H(X \mid Y)+H(Y) \\
& =H(Y \mid X)+H(X) .
\end{aligned}
$$

Since $H(X)$ represents the uncertainty in $X$ before we know $Y$, and $H(X \mid Y)$ represents the uncertainty after, the difference $H(X)-H(\mathrm{X} \mid \mathrm{Y})$ must represent the amount of information provided about $X$ by $Y$. This quantity is called the mutual information between $X$ and $Y$ and is formally given by

Therefore,

$$
\begin{aligned}
I(X, Y) & =\sum_{x, y} P(x, y) \log \left(\frac{P(x, y)}{P(x) P(y)}\right) \\
& =H(X)-H(X \mid Y) \\
& =H(Y)-H(Y \mid X) \\
H(X, Y) & =H(X)+H(Y)-I(X, Y) .
\end{aligned}
$$

Figure 3 shows interrelationship between these entropies. In the graphical representation of a cause and effect network structure, a non-negative branch weight $l(x, y)$ is assigned to every branch of the tree.

\section{$H(X, Y)$}

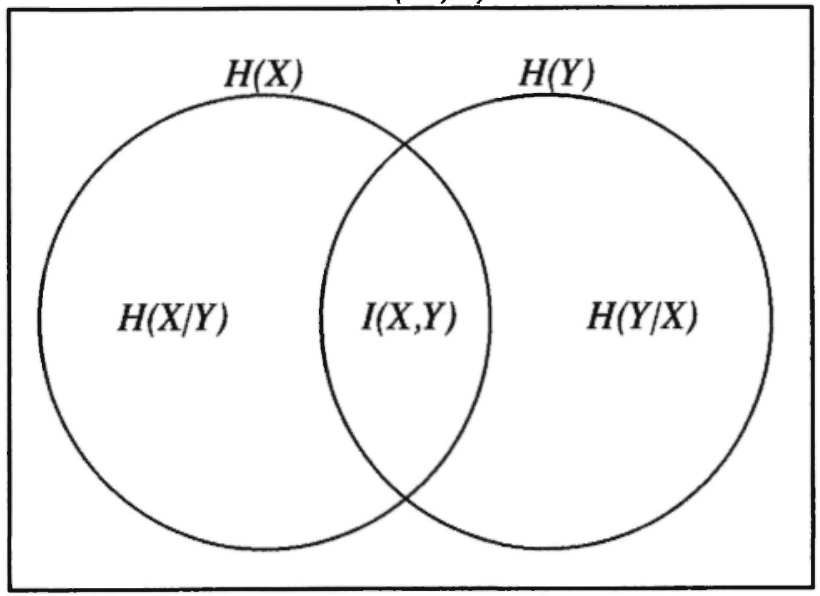

Fig. 3: A diagrammatic representation of the interrelationships between the entropies and mutual information. 
We use this method for the purpose of finding the maximum-likelihood estimator of the distribution of the tree dependence. The structure of the algorithm is shown below.

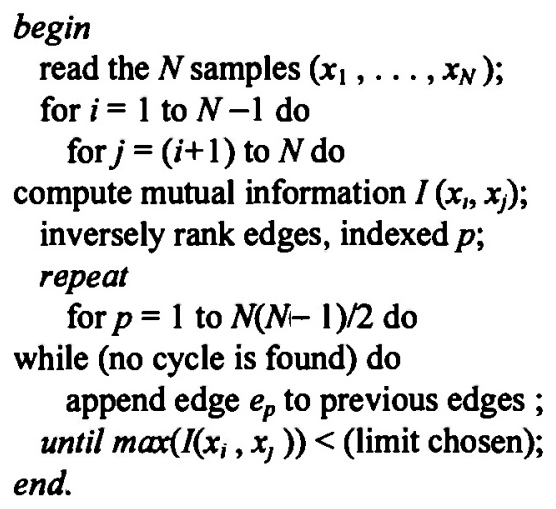

\subsection{The Rough Set Algorithm (RS)}

Rough set theory is another mathematical tool that deals with uncertainty in knowledge bases (Pawlak, 1982; 1991). This method overlaps with several other methods such as Dempster-Shafer theory of evidence (Skowran \& Grzymala-Busse, 1994), or fuzzy set methods (Dubois \& Prade, 1990). These two methods require external parameters but rough set analysis avoids preliminary or additional information about data, such as probability distribution in statistics. Instead, it only uses the structure of the given data, which is a strong advantage when the knowledge available is too vague.

Basic concepts of rough sets. An equivalence $\theta$ on a universe set $U$ is a transitive, reflexive, and symmetric binary relation. The pair $V=\langle U, \theta\rangle$ is an approximation space. The relation $\theta$ is called an indiscernible relation, normally associated with a set of attributes $\left\{x_{1}, \ldots, x_{n}\right\}$. Our knowledge about a subset $Z$ of $U$ is also limited to the classes of $\theta$. However, rough set theory has a tool to deal with inconsistencies. This leads to the definition of $\theta$-lower approximation $\left(Z^{\theta-}\right)$ and $\theta$-upper approximation $\left(Z^{\theta+}\right)$ of $Z$.

$$
Z^{\theta^{-}}=\bigcup_{x_{i}^{\theta} \subseteq Z} x_{i}^{\theta} \quad Z^{\theta^{*}}=\bigcup_{x_{i}^{\theta} \cap Z \neq \theta} x_{i}^{\theta}
$$


It is understood that there are three distinct regions for a subset $Z \subset U$ :

1. $Z^{\circ}$ is called the positive region of $Z$ in $V$;

2. $Z^{\theta^{+}}-Z^{\theta-}$ is called the boundary region of $Z$ in $V$;

3. $U-Z^{\theta^{+}}$ is called the negative region of $Z$ in $V$.

The $\theta$-lower approximation (or positive region) of $Z\left(Z^{\dagger}\right)$ is the collection of objects that can be classified with full certainty as members of the set $Z$, using knowledge $\theta$. This is the greatest definable set in $\theta$ containing $Z$. Similarly, the negative region is the collection of objects with which it can be determined without any ambiguity, employing knowledge $\theta$, that they belong to $-Z$ which denotes the complement $U-Z$ of $Z$. The borderline region (or boundary region) is the undecidable area of the universe $U$ within which objects cannot be classified with certainty into $Z$ or $-Z$. The least definable set in $\theta$ containing $Z$ is the set $Z^{0}$. This set consists of objects with which knowledge $\theta$ does not allow us to exclude the possibility that those objects may belong to $Z$.

Given a partition $U / x_{j}$ in universe $U$, we define the support subset in $U$ for $x_{j}$ from $x_{i}$ as

$$
S_{x_{1}}\left(x_{\jmath}\right)=\bigcup_{Z \in U / x_{j}} Z^{x_{i}^{-}}
$$

The support degree from $x_{1}$ to $x_{j}$, which is defined by

$$
s p t_{x_{i}}\left(x_{j}\right)=\frac{\sum_{Z \in U / x_{j}}\left|Z^{x_{i}^{-}}\right|}{|U|}
$$

is a measure of quality of approximation of the partition $x_{j}$ by set $x_{1}$ of attributes. The values of this measure range between 0 and 1 , where

$$
\begin{aligned}
& s p t_{x}\left(x_{j}\right)=1 \Rightarrow x_{j} \text { is totally dependent on } x_{1} \text { (ie } x_{1} \text { causes } x_{j} \text { ) } \\
& 0<t_{x_{i}}\left(x_{j}\right)<1 \Rightarrow x_{j} \text { roughly depends on } x_{1} \\
& s p t_{x_{i}}\left(x_{j}\right)=0 \Rightarrow x_{1} \text { and } x_{j} \text { are totally independent. }
\end{aligned}
$$

In Table 1 attributes $x_{3}$ and $x_{4}$ define the following elementary sets :

$$
\begin{array}{ll}
Z_{31}=U /\left\{x_{3}=1\right\}=\left\{u_{7}, u_{8}, u_{9}\right\} & Z_{32}=U /\left\{x_{3}=0\right\}=\left\{u_{1}, u_{2}, u_{3}, u_{4}, u_{5}, u_{6}\right\} \\
Z_{41}=U /\left\{x_{4}=1\right\}=\left\{u_{4}, u_{6}, u_{8}, u_{9}\right\} & Z_{42}=U /\left\{x_{4}=0\right\}=\left\{u_{1}, u_{2}, u_{3}, u_{5}\right\}
\end{array}
$$


The support subset for $x_{3} \stackrel{\text { causes }}{\longrightarrow} x_{4}$ is

$$
\begin{aligned}
S_{x_{3}}\left(x_{4}\right) & =\underset{Z=Z_{41}, Z_{42}}{\bigcup} Z^{x_{3}^{-}} \\
& =Z_{41}^{x_{3}^{-}} \cup Z_{42}^{x_{3}^{-}} \\
& =\left(\underset{Z=Z_{31}, Z_{32} ; Z \subseteq Z_{41}}{\bigcup} Z\right) \cup\left(\bigcup_{Z=Z_{31}, Z_{32} ; Z \subseteq Z_{42}}^{\bigcup} Z \begin{array}{r}
U_{31} \\
\end{array}\right) \\
& =Z_{31} \cup \varnothing=Z_{31} .
\end{aligned}
$$

The strength of this dependency is

$$
s p t_{x_{j}}\left(x_{4}\right)=\frac{\sum_{Z \in U / x_{4}}\left|Z^{x_{3}-}\right|}{|U|}=\frac{\left|Z_{31}\right|}{|U|}=\frac{39}{71}
$$

To complete the calculation for the Bayesian and effect network edge $x_{5}-x_{4}$ we need to calculate the support subset for

$$
x_{4} \stackrel{\text { couses }}{\longrightarrow} x_{3} \text { and } s p t_{x_{4}}\left(x_{3}\right) \text {. }
$$

The output of this computation is

$$
S_{x_{4}}\left(x_{3}\right)=\varnothing \cup Z_{42}=Z_{42}
$$

and

$$
\operatorname{spt}_{x_{4}}\left(x_{3}\right)=\frac{\sum_{\mid Z \in U / x_{4}}\left|Z^{x_{3}-}\right|}{|U|}=\frac{\left|Z_{31}\right|}{|U|}=\frac{22}{71}
$$

The causal support of $x_{j}$ from $x_{i}\left(S T R_{x_{j}}\left(x_{j}\right)\right.$ is

$$
\operatorname{STR}_{x}\left(x_{j}\right)=s p t_{x_{j}}\left(x_{j}\right)+s p t_{x_{j}}\left(x_{t}\right)=\frac{\left|Z_{i 2}\right|+\left|Z_{j 1}\right|}{|U|}
$$

This supports the conclusion that Thermostat Flip $\left(x_{4}\right)$ causes Warm Water $\left(x_{3}\right)$ with the strength $(22 / 71+39 / 71)=61 / 71$. 
The pseudocode for the RS algorithm is as follows:

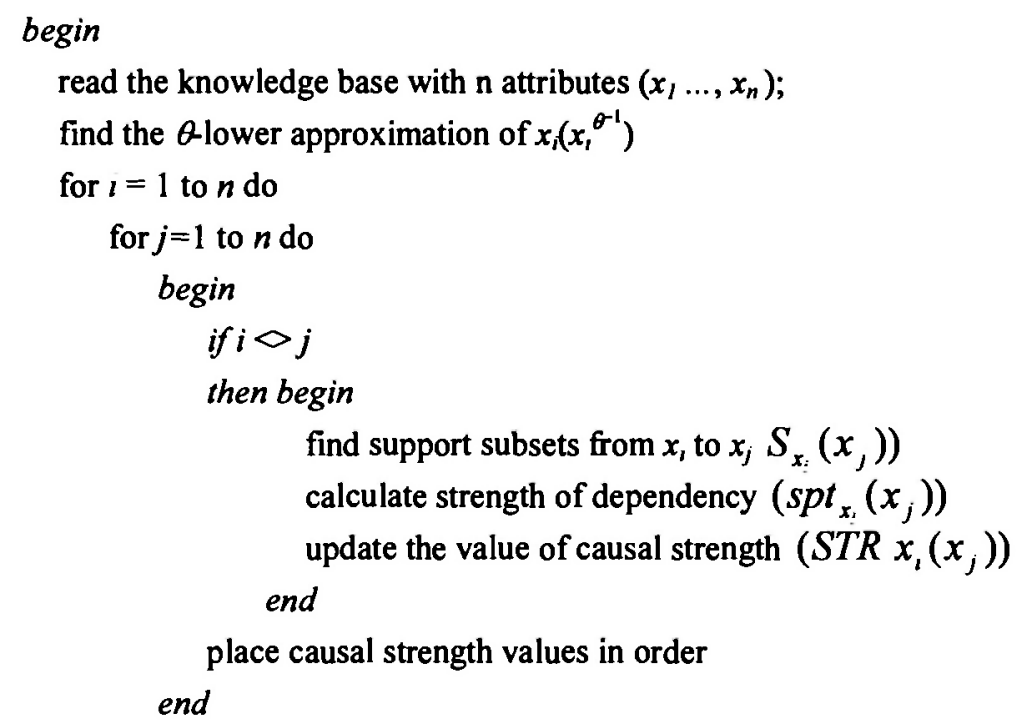

end.

\subsection{The Heuristic Network Generation Algorithm (HNG)}

Another algorithm which we are currently developing is the $H N G$ algorithm. The key steps of this heuristic algorithm, in order of priority, which we developed ourselves for our application are shown below.

\section{begin}

After inputting a "cleaned" relation $(R)$ use simple counts to establish strengths of linkages from co-occurrences of nodal attributes;

Prune the graph using the following heuristics consecutively;

H1 If both nodes are exogenous (ie occur by themselves in tuples of $R$ ), then omit the edge;

H2 If a path including the heuristic edge is "transitively equivalent" to another edge, then omit the smaller edge;

$\mathrm{H3}$ "Singletonise" causes of a node by choosing the direction of edges or removing weak edges to ensure that only one cause remains;

H4 If one node of an edge is exogenous, 
then it is the cause of the other node ;

H5 If a few possible final structures exist then choose the final deliverance which maximises the "out fan" from the exogenous node(s);

H6 Any "precedence evidence" overwhelms any "exogenous evidence" (i.e. variable occurs by itself in structure) regardless of magnitude. e.g. If $p(A \mid 0)<p(B \mid 0)$ (exogenous evidence) AND $p(A \mid B)<p(B \mid A)$ (precedence evidence) Then the evidence $\mathrm{A} \rightarrow \mathrm{B}$ is more valuable.

end.

This algorithm gave promising results in the early stages of the present study and we hope to made further improvements to it in the future.

\subsection{The CAEGA Algorithm}

Our cause and effect genetic adaptation algorithm (CAEGA) is a search process, based on the mechanics of natural selection and natural genetics (Goldberg, 1989). Genetic algorithms improve over the local scope of other search methods, some of which are gradient-based, by searching in parallel many subspaces in multidimensional spaces with complex topologies. Random search algorithms simply lack the efficiency of genetic algorithms.

In genetic algorithms the search space of the problem is represented as a collection of individuals. The latter are represented by character strings, which are often referred to as chromosomes. The part of the search space to be examined is called the population. A genetic algorithm (GA) operates through a simple cycle of four stages:

1. Creation of a population of strings;

2. Evaluation of each string;

3. Selection of best strings; and

4. Genetic manipulation to create the new population of strings.

Figure 4 shows these four stages. Each cycle produces a new generation of possible solutions for the optimal cause and effect network structure.

The basic structure of $C A E G A$ is shown below. (CAEGA I and $C A E G A$ II are two 'flavors' of this algorithm, where the variables are not ordered and ordered, respectively). 


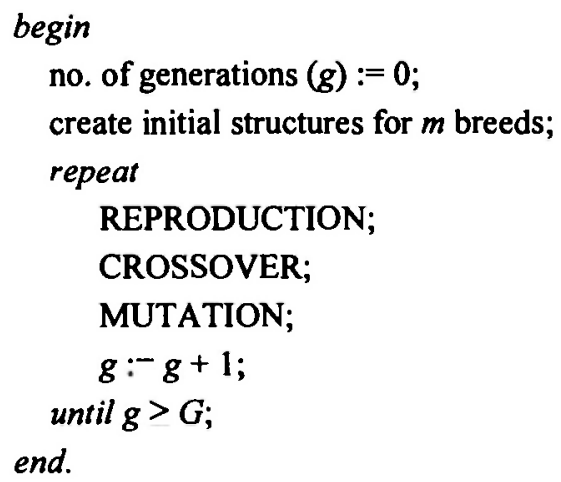

After randomly choosing an initial population, the quality of each of its individuals is determined using the objective 'score' function, based on Cooper's formula (Cooper \& Herskovits, 1992). In REPRODUCTION m/2 pairs of the 'best' breeders (i.e., structures with highest scores) are randomly selected with bias to 'survive' and breed in the next generation. The CROSSOVER

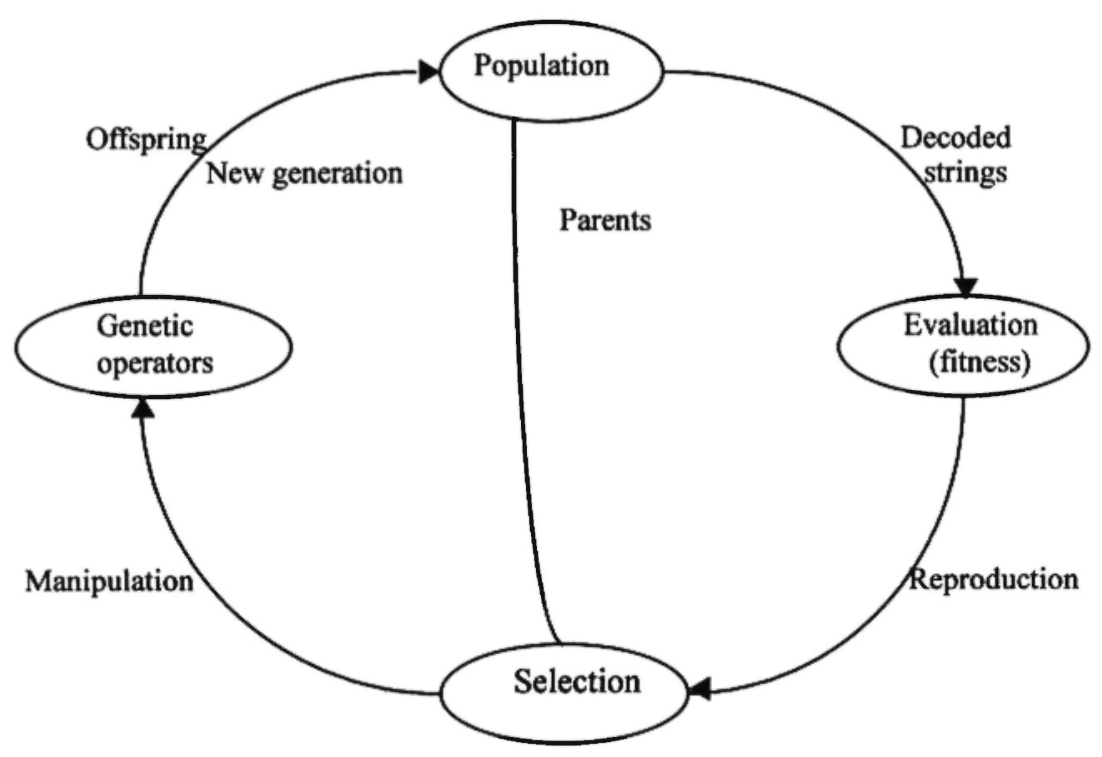

Fig. 4: The GA (genetic algorithms) cycle 
operator produces new offspring structures by transferring a randomly chosen string of fixed length of connectivity elements from one individual to the identical location of its matched pair. A partially mapped crossover (PMX) method was used in our experiments (Goldberg, 1989). MUTATION is an operator that plays a secondary role to CROSSOVER by keeping a high scoring structure of nodes together. This helps CROSSOVER to escape from local optima (Whitley, 1987). This algorithm breeds solutions in accordance with the usual genetic adaptation pattern. This is a variation of an algorithm in (Rojas-Guzman \& Kramer, 1993).

\subsection{Model Averaging}

These algorithms have all been implemented and can be run with MKS, in bread-board style. Our wish is to also implement CANEMAS as a standalone system within a distributed, deductive database system, PDDS (Cao et al., 1993). The results of applying these algorithms are the graphs presented in Fig. 5. The variety is interesting and was, in fact, perhaps a little bit disappointing initially. A method, however, of determining the 'best' graph, sometimes called the 'average', would mean that these variations can each contribute to a consensus. And a consensus is often better than a local optimum. We found it very difficult to evaluate the output for the telecommunication application, as no one knows the 'correct' graph, and even then the results of such a study would be application dependent. But generalized techniques can be envisaged. A final model can be selected, by averaging, in a number of ways. For example, if we simply take votes (i.e., the number of edges supported by other results) on the best selection, structure CE1 would be best for our EVENTS exemplar case (and it is quite intuitive).

Attention, we could breed these solutions using genetic adaptation again, or use one of several A.I. approaches to the solution, or we could use the score directly, to get structure CE5 in this example. The graphs were scored using a method developed by Cooper and colleagues (Cooper \& Herskovits, 1992), and the 'suggestions' can be regarded as a series of expert opinions. The result, however, is somewhat non-intuitive!

The method of choosing the best contender is still an open issue, and so our contribution is claimed to be a supplement to the skilled inputs from 


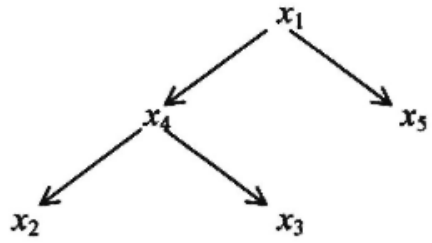

score $(\mathrm{CE} 1)=C^{\star} 1.68012 \mathrm{E}-82$

3. Using rough set approximation approach (CE3)

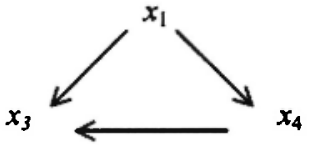

score $(\mathrm{CE3})=\mathrm{C}^{\star} 3.71936 \mathrm{E}-86$

5. Using CAEGA algorithm (not including ordering scheme (CE5)

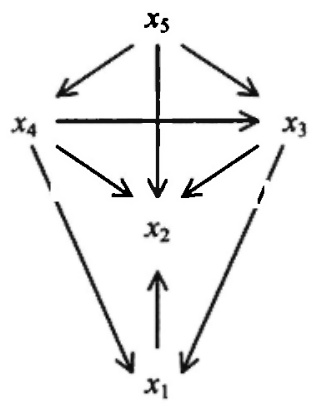

score $($ CE5 $)=c$ * $1.05231 \mathrm{E}-69$

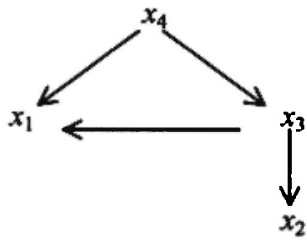

score $($ CE2 $)=C^{\star} 1.70097 \mathrm{E}-82$

4. Using our heuristic algorithm (CE4)

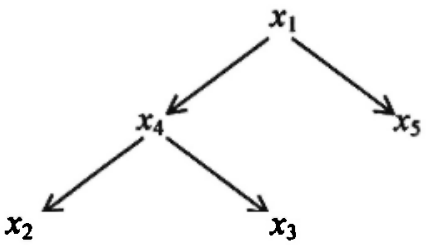

score $(\mathrm{CEA})=\mathrm{C}^{\star} 1.68012 \mathrm{E}-82$

4. Using CAEGA algorithm (including ordering scheme (CE6)

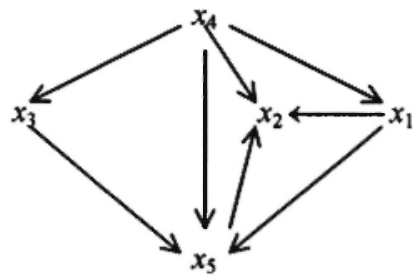

score $($ CE6 $)=c * 1.20136 \mathrm{E}-76$

Fig. 5: Final causal network structure found after running each algorithm. 
domain experts, at the present stage. As we pointed out earlier, supplementation by exogenous domain knowledge was, and is, expected to be indispensable, but clearly quite important inputs to the whole modeling process can be acquired from CANEMAS.

\section{USING GENETIC ALGORITHMS TO GENERATE CAUSAL NETWORKS}

In this section, we show how we applied a rudimentary version of the Cause-And-Effect Genetic Algorithm (CAEGA) to the EVENTS database relation. Table 1 in Sec. 2 shows a projection of this relation over five conventional database attributes. We have added a frequency attribute (Freq) to indicate the number of tuples in the original relation, characterized by each tuple, so we actually have an Augmented Projection operation. Actually the original database here would likely be a conventional relational database of temperatures at different times of day and other data, from which the binary tuples shown are derived in a (clever!) preprocessing step (i.e., even before the Augmented Projection). The sort of causal network that would underpin such a database might intuitively be envisaged as representing relationships, such as a big temperature drop causes both heavy rain and a thermometer flip in a house (see Fig. 6). The latter in turn might cause the given room in the house to warm up and ensure that there is warm water in the household hot-water tank.

As an alternative to $C A E G A$ (or as a preliminary step), the causal ordering of the variables (or attributes) could be obtained by defining the order in which various (subsets of) attributes can be identified (Druzdzel \& Simon, 1993). For example, an attribute, such as $x_{1}$, in various subsets of attributes appear along with others e.g. $x_{4}$ and $x_{5}$ respectively, and if it sometimes appeared by itself, we would conjuncture the top two edges of the graph in Fig. 6. For the purposes of demonstration, we used the resulting 'structural equations' to generate the intuitively plausible graph shown in Fig. 6. The self-explanatory quantitative data in the boxes is readily obtained, for example from the technique suggested in (Cooper \& Herskovits, 1992).

Our probabilistic $C A E G A$ represents the search space of the problem as a collection of individuals. Each individual is represented by a string of 
connectivity elements of an evidential causal network structure (Larranaga et al., 1995). This is written in the form

$$
C E=\left(c_{21} c_{31} c_{32} \ldots c_{i(1-l)} c_{(i+1) l} \ldots c_{n(n-1)}\right)
$$

where

$$
c_{i j}=\left\{\begin{array}{l}
0, \text { if } x_{i} \text { and } x_{j} \text { are independent } \\
1, \text { if } x_{i} \text { is an offspring of } x_{j} \\
2, \text { if } x_{i} \text { is a parent of } x_{j}
\end{array}\right.
$$

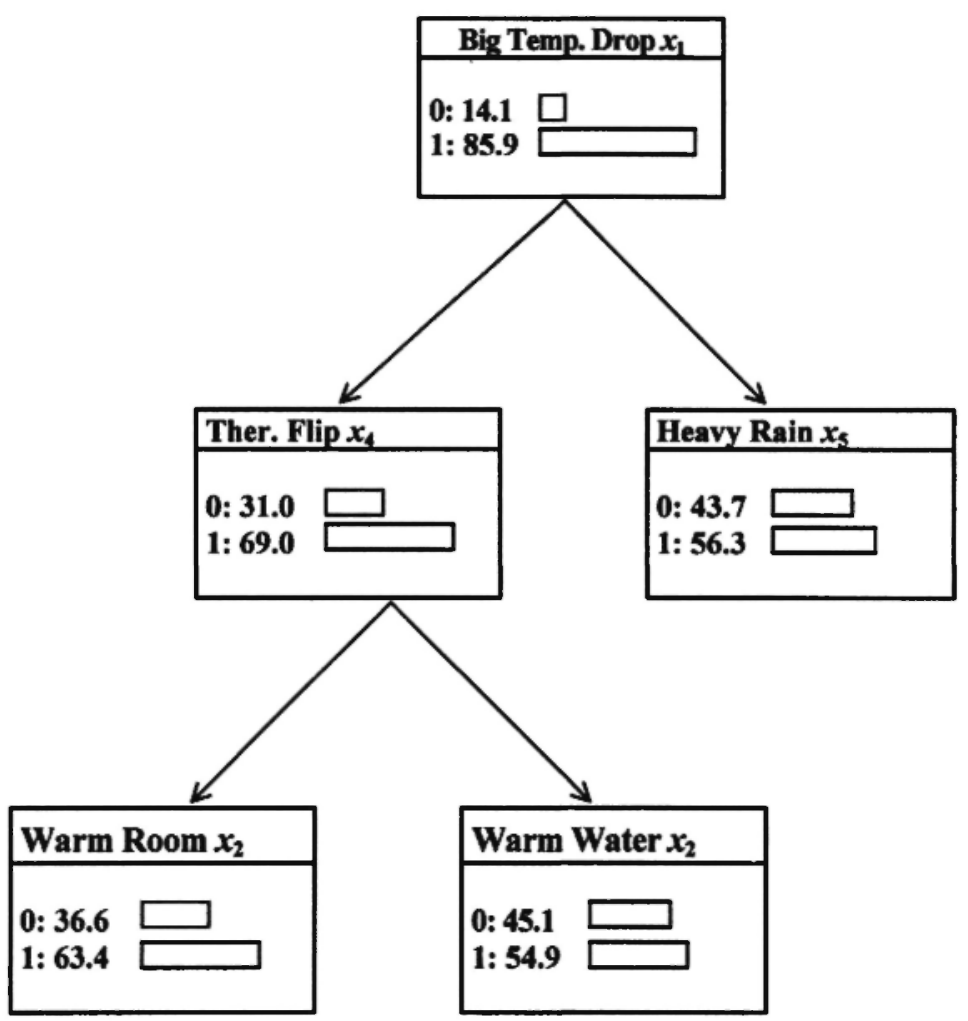

Fig. 6: Optimal causal tree $\left(C E_{1}\right)$ produced by $C A E G A$ for the $E V E N T S$ database. 
Note that this is a slight modification of the signature format proposed in (Larranaga et al., 1995) to account for network structures that are unidirectional and do not assume any ordering of nodes. According to this format, the string that represents the final network structure in Fig. 6 is

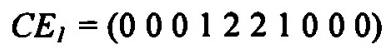

which is illustrated in Fig. 7.

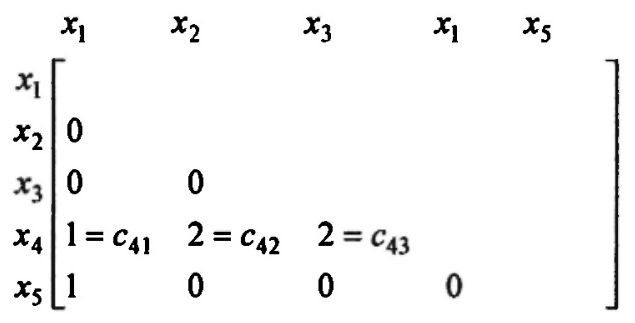

Element $c_{42}$ indicates that $x_{4}$ is a parent of $x$

Fig. 7: $A$ further illustration of the causal network $C E_{1}$

The basic structure of the genetic adaptation algorithm is shown below. (Refer to Fig. 8 for illustration.)

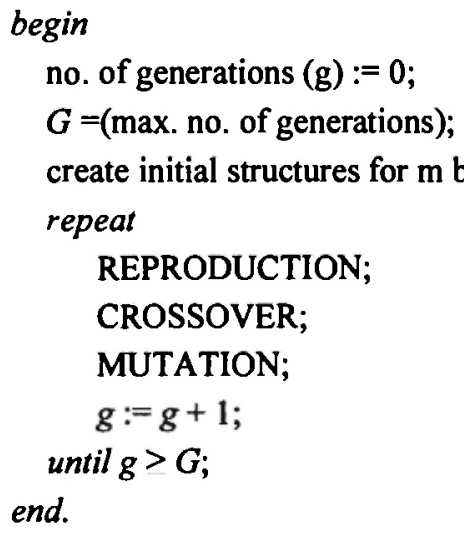

After randomly choosing an initial population, the quality of each of its individuals is determined using the objective "score" function, based here on 
Cooper's formula (Cooper \& Herskovits, 1992). In REPRODUCTION pairs (five in our example), of the 'best' breeders (highest in scoring) are randomly selected with bias to 'survive' and breed in the next generation. The CROSSOVER operator produces new offspring structures by transferring a randomly chosen string of fixed length of connectivity elements from one individual to the identical location of its matched pair. A partially mapped crossover (PMX) method was used in our experiments (Goldberg, 1989). This operator maps a portion of one parent string onto a portion of the other parent string and the remaining information is exchanged. Consider, for example, the two parents of the causal network $C E_{1}$ at generation $310\left(C E_{17}\right.$ and $\left.C E_{14}\right)$ as shown in Fig. 8. The PMX operator begins by selecting at random two cut points along the strings which represent the parents. In this demonstration, a fixed string of three connectivity elements was chosen for each CROSSOVER procedure as shown below, between the third and fourth elements, and the sixth and seventh elements.

$$
C E_{17}=(000|121| 1000) \text { and } C E_{14}=\left(\begin{array}{lll}
0 & 0 & 0|122| 0002
\end{array}\right)
$$

The substrings between the cut points are called the mapping sections. After swapping the two mapping sections, which contain elements $c_{41}, c_{42}$, and $c_{43}$ (indicated in Fig. 7), the two offspring produced are :

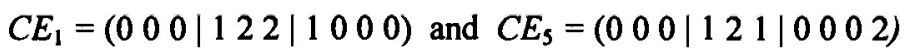

This produces the optimal structure $C E_{1}$, shown in Fig. 6, which is an intuitively acceptable structure. ( $C E_{5}$ is not displayed.)

MUTATION is an operator that plays a secondary role to CROSSOVER by keeping a high scoring structure of nodes together. This helps CROSSOVER to escape from local optima (Whitley, 1987). After selecting a substring at random this substring is removed and inserted in a random place as shown below.

$$
C E_{x}=(110|002| 0010)
$$

and

$$
C E_{x}^{\prime}=(11000|002| 10) .
$$

When we executed a rudimentary version of our CAEGA algorithm with the EVENTS database, the optimal causal network structure produced when the method (Druzdzel \& Simon, 1993; Simon, 1953). The last three generations of the 
GEN. 309

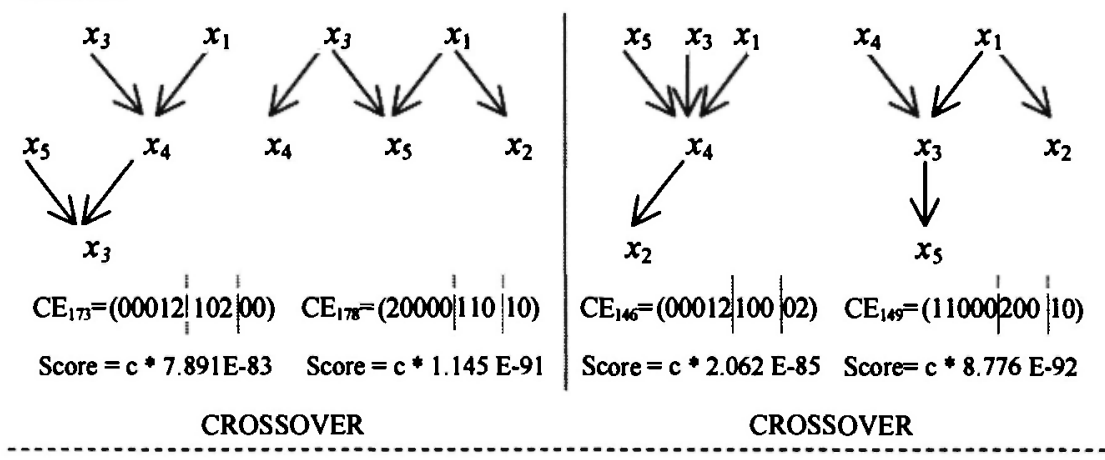

GEN. 311
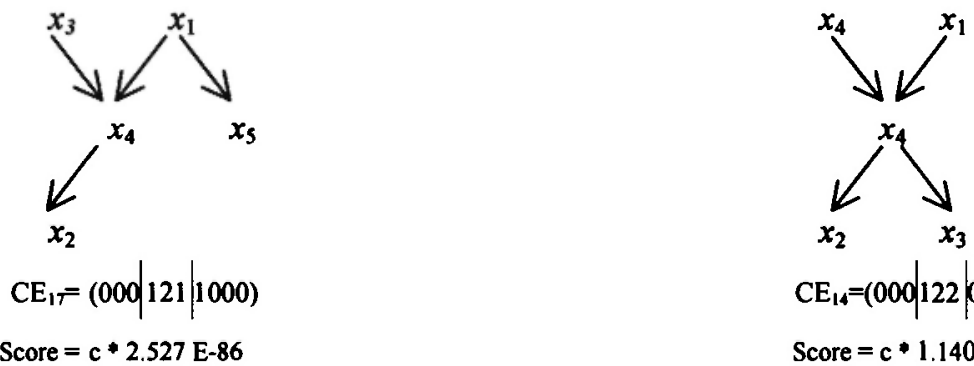

$\mathrm{CE}_{14}=(000 \mid 122$ p002)

Score $=c * 1.140 \mathrm{E}-84$

\section{CROSSOVER}

GEN. 311

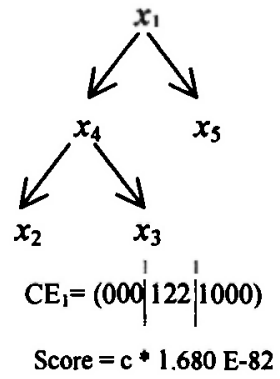

Fig. 8: The final 3 generation of the $C A E G A$ algorithm that produced $C E_{1}$ 
termination criterion was reached agreed with that produced by Simon's simulation that led to the optimal network shown in Fig. 6 are shown in Fig. 8. At generation 309, elements $c_{43}, c_{51}$, and $c_{52}$ swap places in each matched pair. Two structures produced from this generation are the parents of Fig. 6 at generation 310 .

\section{AN OVERVIEW OF THE DEMPSTER-SHAFER THEORY}

After a causal network has been generated, new data may be observed at a later stage. The data might change the causal relationships in our generated network, either because the original network didn't model reality well enough or because the modeled ontology is changing and, therefore, the model has to be adjusted. Pure Bayesian theory has been used extensively to keep causal networks in equilibrium in dynamic systems (Cheng et al., 1997; Neapolitan, 1990; Pearl, 1988). ability to model the narrowing of the hypothesis set with the accumulation of evidence and deals explicitly with ignorance, a portion of our belief that we do not want to allocate to any given hypothesis (Gordon \& Shortliffe, 1984; Shafer, 1976). We recently developed the PropBel (PropagationBelief) algorithm for causal updating, based on this evidential approach.

The Dempster-Shafer theory is based on the idea of allocating a number between 0 and 1 to indicate the degree of belief of evidence for a hypothesis or a proposition (Shafer, 1976). The theory also supports reasoning that is based on the rule of combination $(\oplus)$ by which degrees of belief that are based on different pieces of evidence can be combined. The frame of discernment, denoted by $\Theta$, is a finite nonempty set of propositions that are mutually exclusive and exhaustive. Our experiments were done using binary databases (i.e., where each attribute value is 0 or 1$)$. Therefore the frame of discernment used in this paper is $\Theta-\{0,1\}$.

A mass function $(\mathrm{m})$ is a basic probability assignment function where $m: 2^{\Theta} \rightarrow[0,1]$ such that

$$
m(\varnothing)=0 \quad \text { and } \quad \sum_{\chi \subset \Theta} m(\chi)=1 .
$$

A mass function is assigned to every subset of $\Theta$ such that the numbers sum to 1 . To allow for cases corresponding to the description of the real world, the 
quantity $m(\Theta)$ is a measure of the portion of total belief that remains unassigned after committing ignorance of belief to various subsets of $\Theta$. For example, not all evidence may have been provided to form a causal network. Thus, the remaining belief, the ignorance, is assigned to $\Theta$, so that

$$
\begin{aligned}
& m(\Theta) \sum_{\chi \subset \Theta} m(\chi) . \\
& m(\Theta)=1-\sum_{\chi \subset \Theta} m(\chi) .
\end{aligned}
$$

If $m_{1}(\chi)$ and $m_{2}(\chi)$ are two mass functions included by two independent (see Sec. 7) evidential sources, then the combined mass function is calculated according to Dempster's rule of combination which is

$$
\begin{aligned}
& \left(m_{1} \oplus m_{2}\right)(\chi)=\alpha \sum_{A \cap B=\chi} m_{1}(A) m_{2}(B) \\
& \text { where } 1 / \alpha=1-\sum_{A \cap B=\varnothing} m_{1}(A) m_{2}(B)
\end{aligned}
$$

The normalisation constant $(\alpha)$ is required to make the new quantity a mass function (i.e., summing to 1 , by definition of a mass function). The operator $\oplus$ is also known as the orthogonal sum, and it can be readily extended to the combination of $\mathrm{n}$ mass functions.

For example, suppose $\Theta=\{0,1\}$ and

$$
m_{1}(\{0\})=0.4, \quad m_{1}(\{1\})=0.5, \quad m_{1}(\{0,1\})=0.1 ;
$$

and

$$
m_{2}(\{0\})=0.7, \quad m_{2}(\{1\})=0.2, \quad m_{2}(\{0,1\})=0.1
$$

the orthogonal sum of these two mass functions is shown below. (Conveniently represented as an intersection table.)

\begin{tabular}{llll}
$m_{1} \oplus m_{2}$ & $\mid\{0\}_{0.7}$ & $\{1\}_{0.2}$ & $\{0,1\}_{0.1}$ \\
\hline$\{0\}_{0.4}$ & $\mid\{0\}_{0.28}$ & $\varphi_{0.08}$ & $\{0\}_{0.04}$ \\
$\{1\}_{05}$ & $\mid \varphi_{0.35}$ & $\{1\}_{0.10}$ & $\{1\}_{0.05}$ \\
$\{0,1\}_{0.1}$ & $\mid\{0\}_{0.07}$ & $\{1\}_{0.02}$ & $\{0,1\}_{0.01}$
\end{tabular}

Here, each subset $\chi$ appears only once and the empty set $\varphi$ never appears so that $\left(m_{1} \oplus m_{2}\right)(\chi)$ is easily computed without the need for $\alpha$ to be calculated. 


$$
\begin{aligned}
& \left(m_{1} \oplus m_{2}\right)(\{0\})=(0.28+0.04+0.07) /(1-0.43)=0.68 \\
& \left(m_{1} \oplus m_{2}\right)(\{1\})=(0.10+0.05+0.02) /(1-0.43)=0.30 ; \text { and } \\
& \left(m_{1} \oplus m_{2}\right)(\{0,1\})=0.01 /(1-0.43)=0.02
\end{aligned}
$$

\section{CAUSAL EVIDENTIAL REASONING USING DEMPSTER-SHAFER THEORY}

As indicated earlier, many causal networks are structured in the form of causal trees, in which each variable is limited to more than one parent, as shown in Fig. 2a. Although this restriction simplifies computations, its accuracy for representing causal factors is reduced because to employ it, lots of the nodes may have to be grouped together into a single node. When, for example, the scope of the model is increased and more areas of knowledge are focused upon, further independent variables may be used in the network, and some propositions may then have multiple causes. For example, in the medical world, more than one disease may share a particular symptom. This type of network is known as a causal polytree (see Fig. 2b).

The theory of causal polytrees is an extension of that for causal trees. Both theories share the requirement that they must singly connected (i.e., no more than one path allowed between any two variables). If this feature is ignored, then evidence may circulate indefinitely around the existing loops, thereby preventing the state of equilibrium to be reached. Loops, however, can occur in causal networks and must be removed if polytree methods are to be used. The three basic methods of coping with a loop in a network are clustering, conditioning, and stochastic simulation. Clustering, the method that we favor; is one of the simplest approaches for dealing with this problem of non-singly connected networks. Figure 9a indicates that variable $A$ is an indirect cause of variable $D$ in two different ways. Clustering works by collapsing nodes into a single compound variable, as shown in Fig. $9 \mathrm{~b}$.

In conditioning, the communication pathways along the loops are broken by instantiating a selected group of variables. For example, in Fig. 9a, before new evidential data are propagated through the causal network, the latter is broken into two separate networks by instantiating the binary variable $A$ for each of its values, as shown in Fig. 9cl and Fig. 9c2 (Neopolitan, 1990). This method 
simplifies the propagation of information through the network but could be computationally expensive.

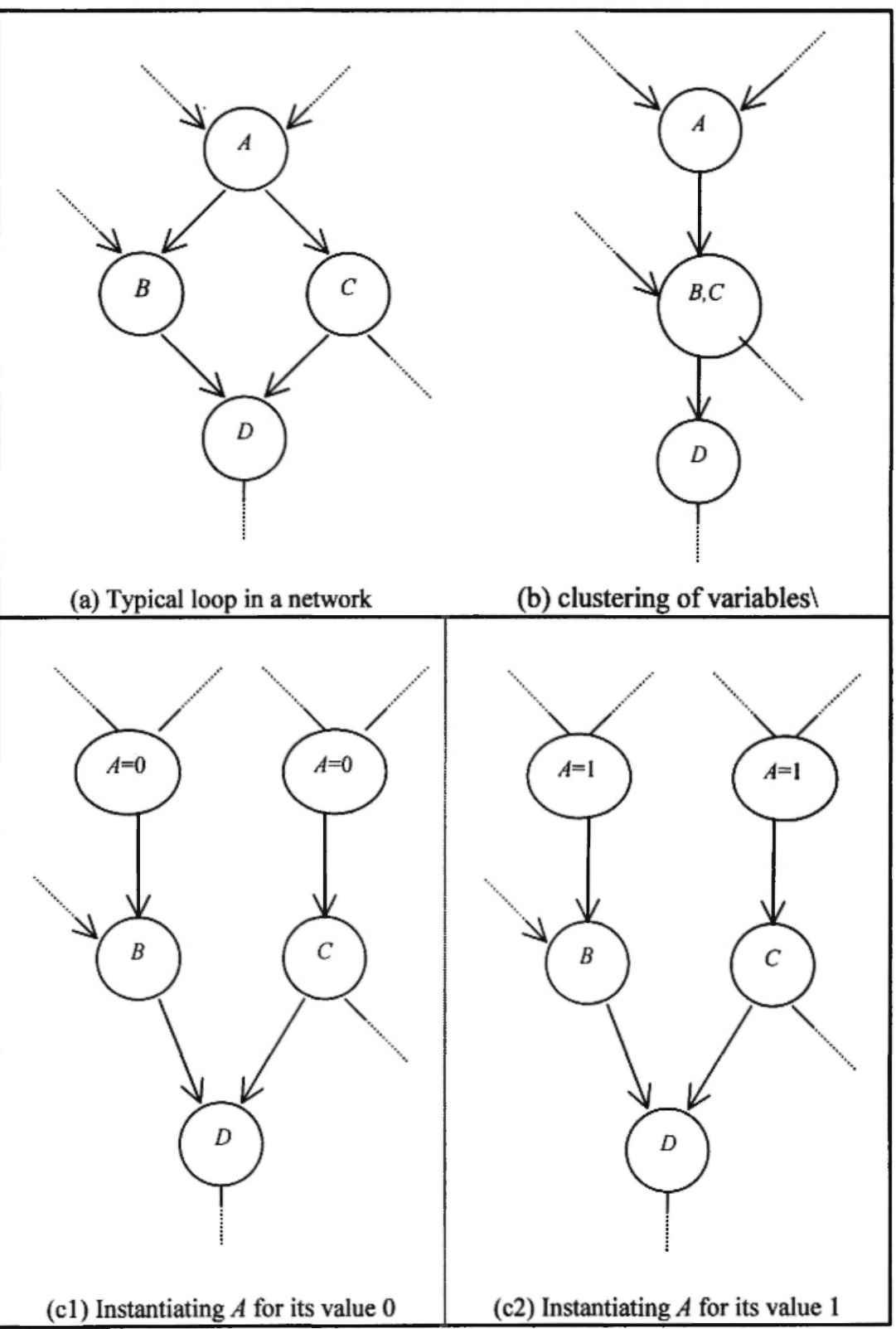

Fig. 9: The clustering and conditioning methods for dealing with non-singly connected causal networks. 
In stochastic simulation, a definite value is assigned to each variable, and each processor inspects the current state of its neighbors. The belief distribution of its host variable is computed, after which one value at random is selected from the computed distribution. This method may require very long simulation times before reaching a proper equilibrium, but convergence is guaranteed. Once a causal network is guaranteed to be singly connected, a reasoning process can begin when any node receives activated data. Belief updating and belief propagating are the two key steps of a reasoning process. As a result of the application of these operations, the nodal belief distributions (examples are shown in Fig. 6) are altered. The following three parameters are made available to accomplish this process:

- Causal support $\left(\mathrm{m}_{\mathrm{P} \downarrow} \mathrm{x}\right)$ contributed by a parent $P$ of $\chi$,

$$
m_{P \downarrow \mathrm{X}}=\left[m_{P \downarrow \mathrm{X}}\left(P_{1}\right), \ldots, m_{P \downarrow \mathrm{X}}\left(P_{l}\right)\right], \quad \text { on } P=\left\{P_{1}, \ldots, P_{l}\right\} .
$$

For example, in Fig. 6 the causal support contributed by $x_{4}$ to $x_{2}$ is

$$
m_{x 4+x^{2}}=\left[m_{x 4 \downarrow \times x 2}(\{0\}), m_{x 4 \downarrow_{x 2}}(\{1\}), m_{x 4 \downarrow_{x 2}}(\{0,1\})\right] \text {, on } x_{4}=\{0,1\} \text {. }
$$

Diagnostic support $\left(m_{C_{i} \uparrow \chi}\right)$ contributed by the $i^{\text {th }}$ child $C_{i}(\forall t=1, \ldots, r)$,

$$
m_{C_{i} \uparrow \chi}=\left[m_{C_{i} \uparrow \chi}\left(X_{1}\right), \ldots, m_{C_{i} \uparrow \chi}\left(X_{\mathrm{w}}\right)\right] \text {, on } \chi=\left\{X_{1}, \ldots, X_{\mathrm{w}}\right\} \text {. }
$$

For example, in Fig. 6 the diagnostic support received by $x_{1}$ from $x_{3}$ is

$$
m_{x s \uparrow x 1}=\left[m_{x \uparrow \uparrow x 1}(\{0\}), m_{x \uparrow \uparrow x 1}(\{1\}), m_{x s \uparrow x 1}(\{0,1\})\right] \text {, on } x_{1}=\{0,1\} \text {. }
$$

- A causal matrix $\left(M_{P \downarrow X}\right)$ or rule strength matrix with $l$ rows and $w$ columns indicating the strength of dependencies between the different values of node $\chi$ and its parent $P$. Each cell $(i, j)$ of the causal matrix is the conditional probability $p\left(\chi=X_{i} \mid P=P_{j}\right)$, which is calculated from the current data representing the link $P \rightarrow X$.

$$
\left(\mathrm{M}_{\mathrm{P} \downarrow \mathrm{X}}\right)=\left[\begin{array}{cccccc}
p\left(X_{1} \mid P_{1}\right) & p\left(X_{2} \mid P_{1}\right) & \ldots & p\left(X_{1} \mid P_{1}\right) & \ldots & p\left(X_{w} \mid P_{1}\right) \\
p\left(X_{1} \mid P_{2}\right) & p\left(X_{2} \mid P_{2}\right) & \ldots & p\left(X_{i} \mid P_{2}\right) & \ldots & p\left(X_{w} \mid P_{2}\right) \\
\ldots & \ldots & \ldots & \ldots & \ldots & \ldots \\
p\left(X_{1} \mid P_{J}\right) & p\left(X_{2} \mid P_{J}\right) & \ldots & p\left(X_{i} \mid P_{j}\right) & \ldots & p\left(X_{w} \mid P_{j}\right) \\
\ldots & \ldots & \ldots & \ldots & \ldots & \ldots \\
p\left(X_{1} \mid P_{l}\right) & p\left(X_{2} \mid P_{l}\right) & \ldots & p\left(X_{i} \mid P_{l}\right) & \ldots & p\left(X_{w} \mid P_{l}\right)
\end{array}\right]
$$


For example, in Fig. 6 the causal matrix for link $x_{4}-x_{3}$, as shown below, is calculated from the EVENTS database in Table 1.

$$
\begin{aligned}
m_{x_{4} \downarrow x_{3}} & =\left[\begin{array}{lll}
p\left(x_{3}=\{0\} \mid x_{4}=\{0\}\right) & p\left(x_{3}=\{1\} \mid x_{4}=\{0\}\right) & p\left(x_{3}=\left\{\Theta \mid x_{4}=\{0\}\right)\right. \\
p\left(x_{3}=\{0\} \mid x_{4}=\{1\}\right) & p\left(x_{3}=\{1\} \mid x_{4}=\{1\}\right) & p\left(x_{3}=\left\{\Theta \mid x_{4}=\{1\}\right)\right. \\
p\left(x_{3}=\{0\} \mid x_{4}=\Theta\right) & p\left(x_{3}=\{1\} \mid x_{4}=\Theta\right) & p\left(x_{3}=\left\{\Theta \mid x_{4}=\Theta\right)\right.
\end{array}\right] \\
& =\left[\begin{array}{lll}
1.0 & 0.0 & 0.0 \\
0.204 & 0.796 & 0.0 \\
0.0 & 0.0 & 1.0
\end{array}\right]
\end{aligned}
$$

It is appropriate to make a statement here about the independence of data. To use the orthogonal sum, $\oplus$, as we do for both updating and propagation, independence must be assured. Now this is a somewhat controversial aspect of Dempster-Shafer theory. (Indeed, it has counterparts in alternative theories also.) For example, if we have a diamond-shape loop like the one in Fig. 9a, clearly if evidence is available at $D$, then belief propagated from $D$ to $B$ and $C$, respectively, might contribute twice to the process of belief accumulation at $A$. Now if we have $B, C$ as a single node (and for example new evidence for either $B$ or $C$ is being treated as evidence for the aggregate), then the question of validity of independence assumptions remains, but is no worse than such assumptions for belief propagation and updating in for example Shafer-Logan's tree structures (or even Bayesian belief networks).

\subsection{Belief Updating}

When a node of a causal network is activated with new evidence, the belief value has to be modified. In turn, this modification affects neighboring nodes so that the updating process continues until the network is completely traversed.

Each node $(\chi)$ computes its own causal support $\left(m_{\downarrow x}\right)$ and diagnostic support $\left(m_{\uparrow_{x}}\right)$. For causal trees this is done by obtaining the causal support of the single parent $P$ and the combined diagnostic support of the children, $C_{1}, \ldots, C_{r}$, where

$$
m_{\downarrow \chi}=m_{P \downarrow \chi} M_{P \downarrow \chi}
$$

and

$$
m_{\uparrow_{\chi}}=\alpha\left(m_{C_{1} \uparrow_{\chi}} \oplus m_{C_{2}} \uparrow \chi \oplus \cdots m_{C_{r} \uparrow_{\chi}}\right),
$$


where $\alpha$ is again a normalisation constant.

The orthogonal sum of these two results is used to update the belief of node $\chi$, given by

$$
\left.m(\chi)=\alpha\left(m_{\downarrow_{\chi}} \oplus\right) m_{\chi}\right) .
$$

The boundary condition for the root node (for example in Fig. 6 this is node $x_{1}$ ) is established by setting

$$
m_{\downarrow \text { root }}- \begin{cases}0, & \text { if } \text { root }=\{0\}, \\ 0, & \text { if } \text { root }=\{1\}, \\ 1, & \text { if root }=\Theta .\end{cases}
$$

(The ignorance here is inherent because we are using the limited representation of the real world that we have available in a database.)

The formulae used for updating beliefs for a causal polytree are similar to those used for a causal tree (McErlean et al., in press; Pearl, 1986), with the exception of the computation of the causal support (because now multiple parents are possible). The message computed to send to node $\chi$ from its multiple parents $\left\{P_{1}, \ldots, P_{v}\right\}$ is

$$
m_{\downarrow_{X}}=\alpha\left(m_{P I \downarrow X} M_{P I \downarrow X} \oplus \cdots \oplus m_{P \downarrow X} M_{P_{\downarrow} \downarrow}\right) .
$$

\subsection{Belief Propagation}

The propagation scheme allows the belief to be distributed throughout the complete causal network by supplying diagnostic support to the parent of each node (bottom-up propagation) and a causal support to each child (topdown propagation).

The bottom-up propagate mass function $\left(m_{\mathrm{x}} \uparrow P\right)$ on each parent $P$ of a node $\chi$ in a causal tree is calculated from the combined diagnostic support received from the children of $\chi$ and the normalized transpose of the causal matrix ( $M_{P \downarrow \chi}^{T}$ ) stored on the link from the parent. The top-down propagation mass function for each child $C_{1}$ of node $\chi\left(m_{\chi} \downarrow c_{1}\right)$ is calculated using the orthogonal sum of the belief of node $\chi$ in combination with the diagnostic support of every other child $C_{\text {f }}$. The formulae for these calculations are shown below. 
Vol. 10, No. 2, 2000

$$
m_{\chi} \uparrow \mathrm{P}=m_{\uparrow \chi} M_{P \downarrow \chi}^{T}
$$

and

$$
m_{\chi \downarrow C_{i}}=\alpha\left(m(\chi) \oplus \sum_{j=1, \cdots, r ; j \neq i} \oplus m_{C_{j} \uparrow}\right)
$$

In a causal polytree the bottom-up propagate mass function for each parent $P$ is

$$
m_{\chi \downarrow P_{j}}=\alpha\left(m(\chi) \oplus \sum_{k=1, \cdots, m ; k \neq i} \oplus m_{P_{k} \downarrow \chi} M_{P_{k} \downarrow \chi}^{T}\right) .
$$

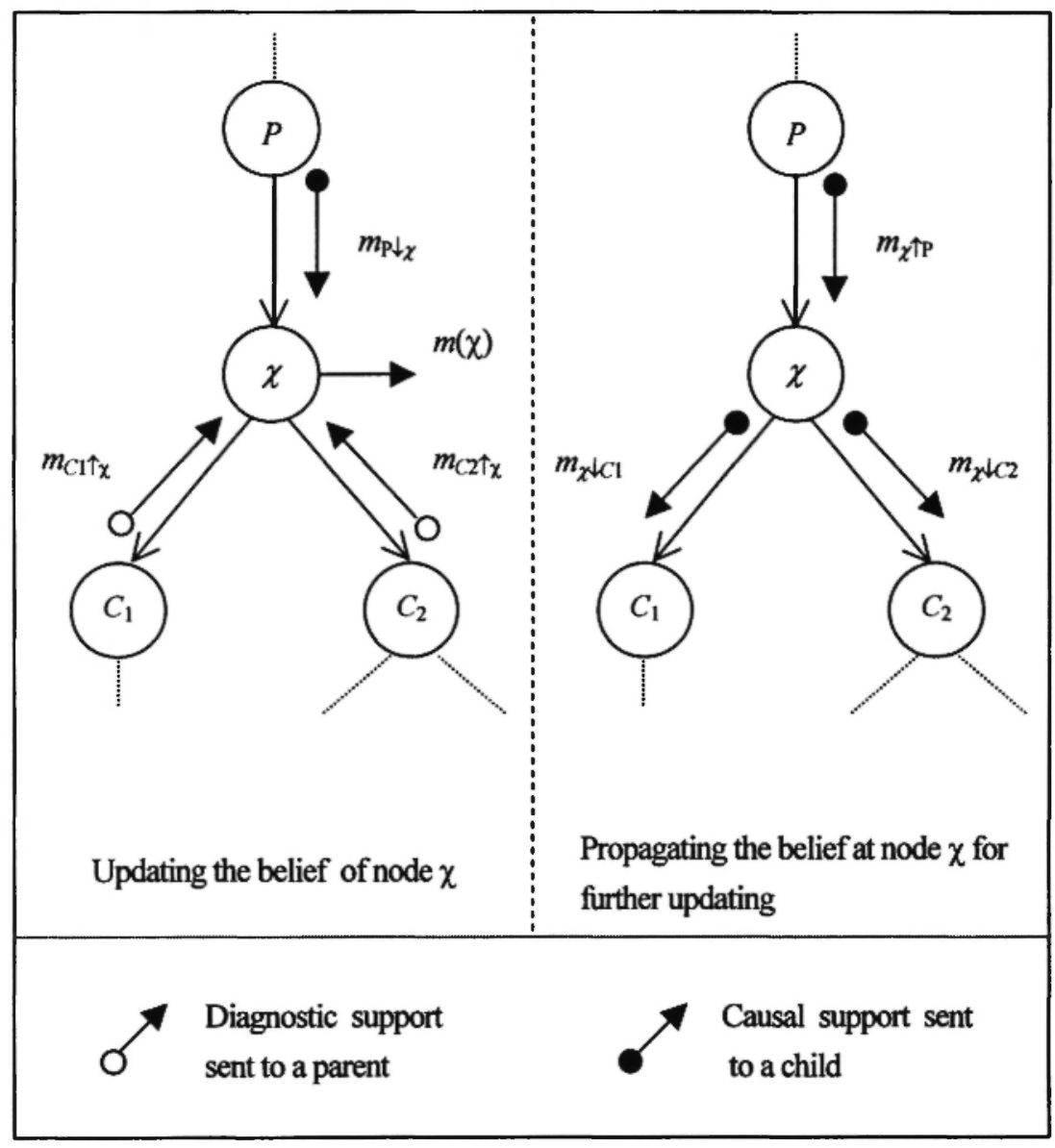

Fig. 10: A fragment of a causal tree showing the updating and propagation of node. 
Figure 10 illustrates belief updating and belief propagation in a tree fragment for a node $\chi$ with children $C_{1}$ and $C_{2}$. These two schemes can be executed in any order.

Figure 11 depicts the four successive states of belief propagation through a simple binary causal tree, assuming that the updating occurs by changes in the belief parameters of neighboring nodes. Initially, the tree is in equilibrium (Fig. 1 la). When data activates on of the leaf nodes, the parent of this node absorbs diagnostic support (see the white token in Fig. 11b).

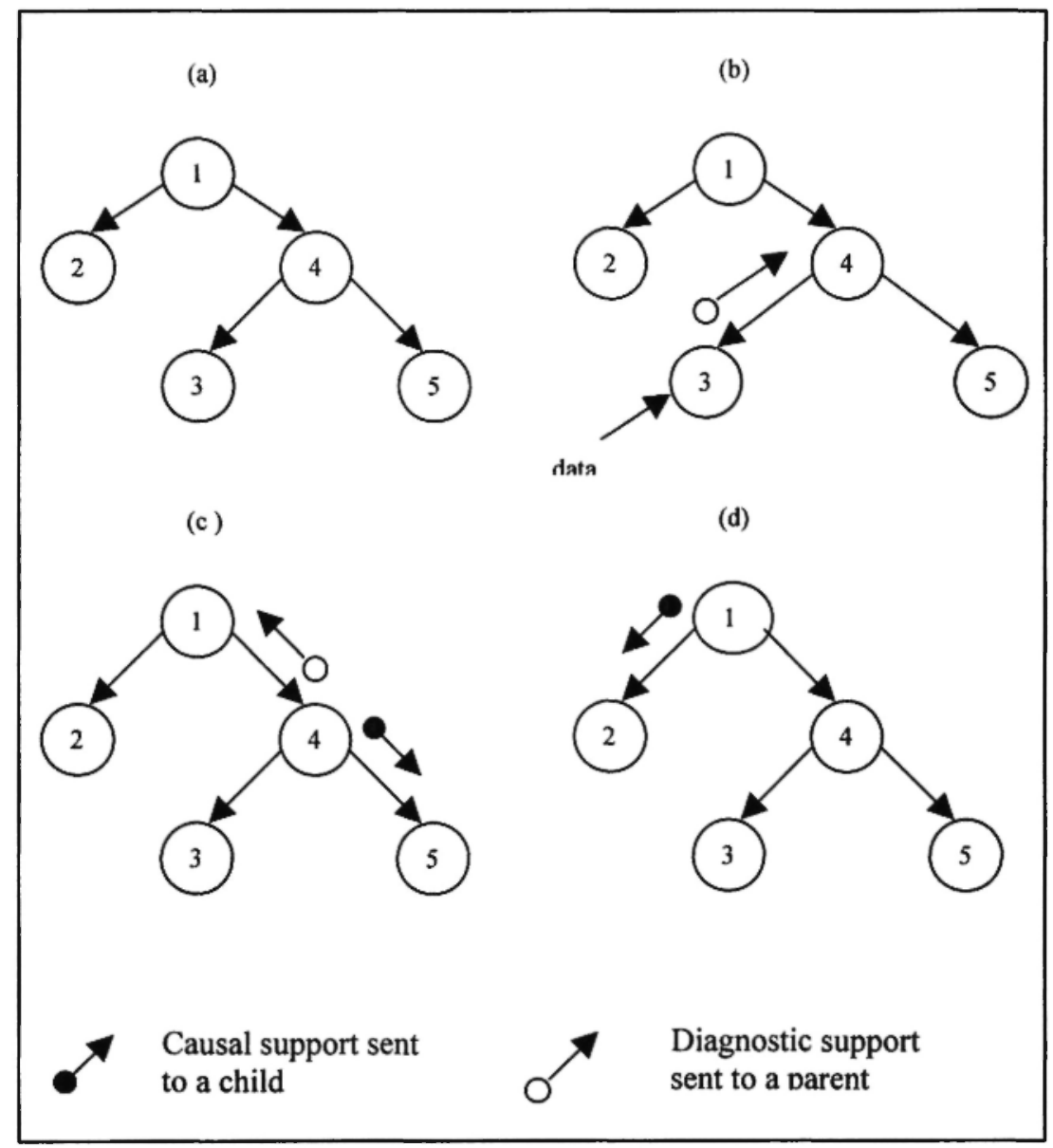

Fig. 11: The three cycles of belief propagation in a 5-node causal tree 
Diagnostic support is passed on to the parent of node 4 for which the belief is updated, and the children of node 4 (apart from node 3) absorb causal support (see the black token in Fig. 11c). Any links from which the absorbed supports originated do not receive updated supports because, for example, $m_{P \downarrow_{X}}$. is not affected by $m_{\chi} \uparrow_{P}$ crossing the same link. The root node then triggers the production of only one black token for a top-down propagation mass function to node 2 (see Fig. 11d). After updating the belief for node 2, the network then reaches a new equilibrium (no further adjustments are available). An example of the application of this algorithm is shown in the following section and in McErlean et al. (in press).

\section{USING PROBEL IN A REAL APPLICATION}

In many domains in the "real world", causal networks can be created to help in a reasoning process, and these have to be regularly kept up to date. New procedures in the medical world often supply new evidence for causal relations between pairs of symptoms that lead to different diseases. When new drugs are supplied, the strength between different interrelations varies. This change calls for the causal network to be kept updated for the purpose of seeing how successful the new drugs are and to help make various choices and new decisions.

The ProBel prototype was used to investigate a telecommunications application. The CAEGA algorithm was used to create a causal network. Further details about this model and the application are discusses in Sterritt et al. (1997a,b). This section focuses upon the evaluation of our ProBel algorithm with the telecommunications data.

A network of multiplexers in a telecommunications industry are used to direct information between different sites to detect various faults. The interactions between components and multiplexers are highly complex. Although an alarm is triggered by each individual multiplexer when a fault occurs, sometimes other alarms occur too, making it more difficutl to find the cause of the fault. We used $C A E G A$ to structure a causal network with the NTELECOM database relation shown in Appendix A (see Fig. 12). The initial values of the support parameters and belief functions for the nodes in this causal polytree are shown in Table 2. 
When new evidence was supplied for node $A_{4}$, the belief function for this node was modified from

$$
m\left(A_{4}\right)=[0.9,0.0,0.1] \quad \text { to } \quad m^{\prime}\left(A_{4}\right)=[0.62,0.25,0.13] .
$$

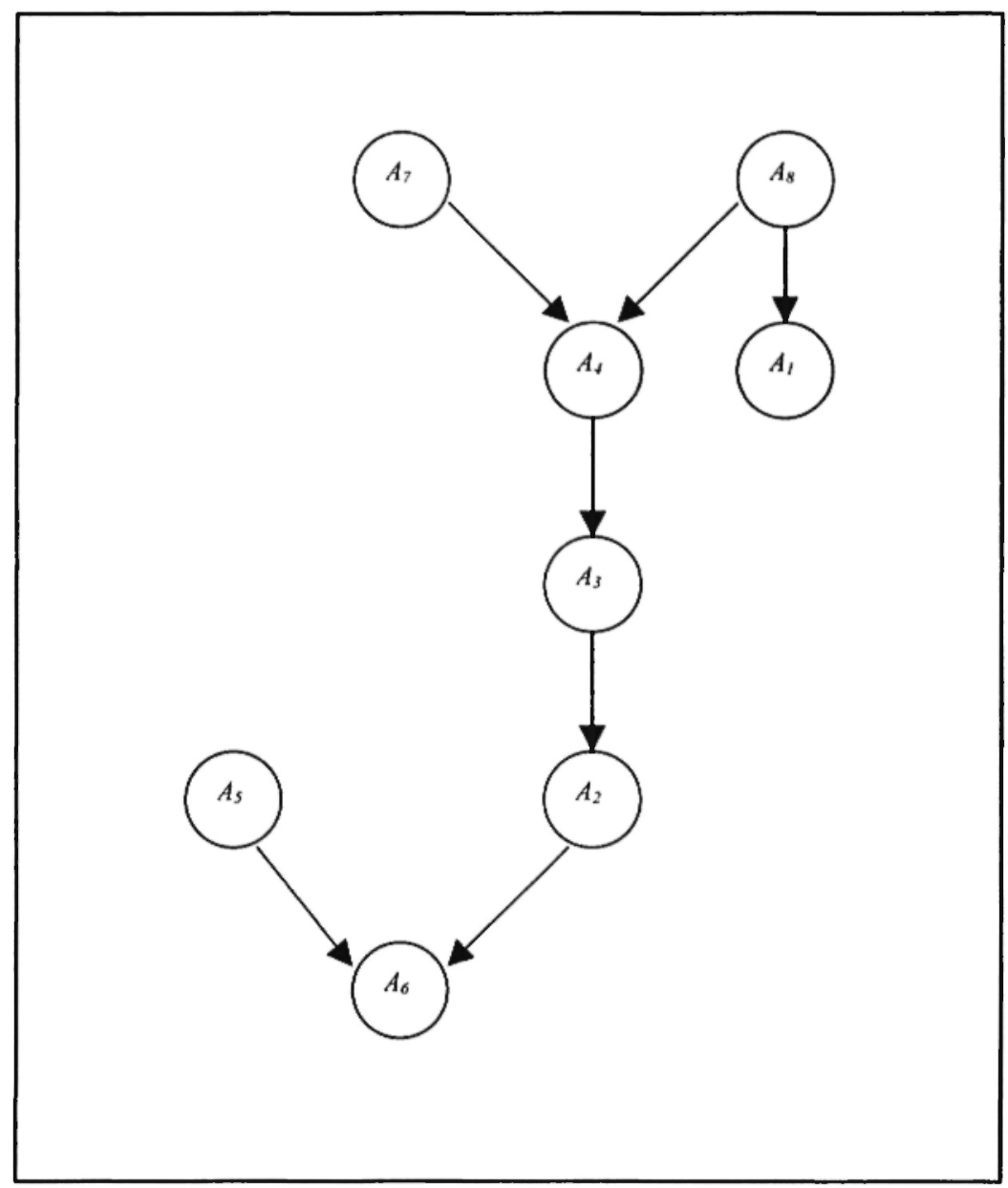

Fig. 12: Optimal causal network structure produced by NETEXTRACT for the NTELECOM database 
TABLE 2

The initial support values of the nodes in the evidential causal network structure representing the NTELECOM relational database

\begin{tabular}{|c|c|c|c|c|c|c|c|c|c|c|}
\hline$\chi$ & $P$ & \multicolumn{3}{|c|}{$m_{P \downarrow_{\chi}}(P)$} & \multicolumn{3}{c|}{$m_{P \uparrow \chi}(P)$} & \multicolumn{3}{c|}{$m(\chi)$} \\
\hline & & $P=\{0\}$ & $P=\{1\}$ & $P=\{0,1\}$ & $P=\{0\}$ & $P=\{1\}$ & $P=\{0,1\}$ & $\chi=\{0\}$ & $\chi=\{1\}$ & $\chi=\{0,1\}$ \\
\hline$A_{1}$ & $A_{8}$ & 0.0 & 0.0 & 1.0 & 0.0 & 0.0 & 1.0 & 0.6 & 0.3 & 0.1 \\
\hline$A_{2}$ & $A_{3}$ & 0.0 & 0.0 & 1.0 & 0.0 & 0.0 & 1.0 & 0.2 & 0.8 & 0.0 \\
\hline$A_{3}$ & $A_{4}$ & 0.0 & 0.0 & 1.0 & 0.0 & 0.0 & 1.0 & 0.6 & 0.4 & 0.0 \\
\hline$A_{4}$ & $A_{7}$ & 0.0 & 0.0 & 1.0 & 0.0 & 0.0 & 1.0 & 0.9 & 0.0 & 0.1 \\
\hline$A_{4}$ & $A_{8}$ & 0.0 & 0.0 & 1.0 & 0.0 & 0.0 & 1.0 & 0.9 & 0.0 & 0.1 \\
\hline$A_{5}$ & - & 0.0 & 0.0 & 1.0 & - & - & - & 0.3 & 0.7 & 0.0 \\
\hline$A_{6}$ & $A_{2}$ & 0.0 & 0.0 & 1.0 & 0.0 & 0.0 & 1.0 & 0.2 & 0.6 & 0.2 \\
\hline$A_{6}$ & $A_{5}$ & 0.0 & 0.0 & 1.0 & 0.0 & 0.0 & 1.0 & 0.2 & 0.6 & 0.2 \\
\hline$A_{7}$ & - & 0.0 & 0.0 & 1.0 & - & - & - & 0.3 & 0.7 & 0.0 \\
\hline$A_{8}$ & - & 0.0 & 0.0 & 1.0 & - & - & - & 0.1 & 0.6 & 0.3 \\
\hline
\end{tabular}

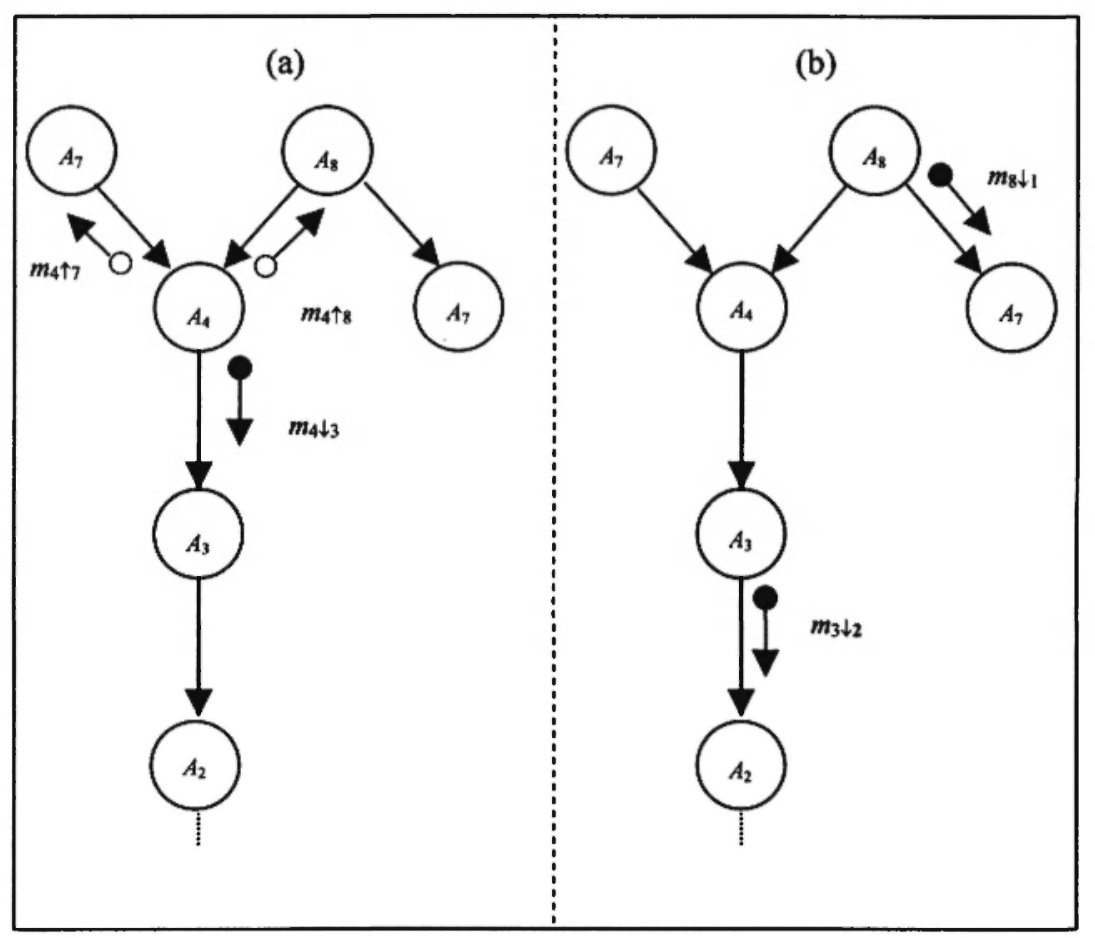

Fig. 13: The first two stages of belief propagation in the causal network for the NTELECOM database 
The first two stages of the evidential flow of new belief propagates through the causal network is shown in Fig. 13. Table 3 displays the updated values of the support mass functions after running our PropBel algorithm. The calculations below show how the support values $m_{A 4 \uparrow_{A B}}$ and $m_{A B \downarrow_{A 1}}$ are updated.

TABLE 3

The updated support values of the nodes after belief propagation for node $A_{4}$.

\begin{tabular}{|c|c|c|c|c|c|c|c|c|c|c|}
\hline$\chi$ & $P$ & \multicolumn{3}{|c|}{$m_{P \downarrow \chi}(P)$} & \multicolumn{3}{c|}{$m_{P \uparrow \chi}(P)$} & \multicolumn{3}{c|}{$m(\chi)$} \\
\hline & & $\mathrm{P}=\{0\}$ & $\mathrm{P}=\{1\}$ & $\mathrm{P}=\{0,1\}$ & $\mathrm{P}=\{0\}$ & $\mathrm{P}=\{1\}$ & $\mathrm{P}=\{0,1\}$ & $\chi=\{0\}$ & $\chi=\{1\}$ & $\chi=\{0,1\}$ \\
\hline$A_{1}$ & $A_{8}$ & 0.791 & 0.185 & 0.024 & 0.00 & 0.00 & 1.00 & 0.687 & 0.288 & 0.024 \\
\hline$A_{2}$ & $A_{3}$ & 0.556 & 0.314 & 0.130 & 0.00 & 0.00 & 1.00 & 0.427 & 0.443 & 0.130 \\
\hline$A_{3}$ & $A_{4}$ & 0.620 & 0.250 & 0.130 & 0.00 & 0.00 & 1.00 & 0.556 & 0.314 & 0.130 \\
\hline$A_{4}$ & $A_{7}$ & 0.000 & 0.000 & 1.000 & 0.62 & 0.25 & 0.13 & 0.620 & 0.250 & 0.130 \\
\hline$A_{4}$ & $A_{8}$ & 0.000 & 0.000 & 1.000 & 0.62 & 0.25 & 0.13 & 0.620 & 0.250 & 0.130 \\
\hline$A_{5}$ & - & 0.000 & 0.000 & 1.000 & - & - & - & 0.819 & 0.156 & 0.025 \\
\hline$A_{6}$ & $A_{2}$ & 0.427 & 0.443 & 0.130 & 0.00 & 0.0 & 1.00 & 0.741 & 0.129 & 0.130 \\
\hline$A_{6}$ & $A_{5}$ & 0.000 & 0.000 & 1.000 & 0.8 & 0.16 & 0.02 & 0.741 & 0.129 & 0.130 \\
\hline$A_{7}$ & - & 0.000 & 0.000 & 1.000 & - & - & - & 0.620 & 0.250 & 0.130 \\
\hline$A_{8}$ & - & 0.000 & 0.000 & 1.000 & - & - & - & 0.620 & 0.250 & 0.130 \\
\hline
\end{tabular}

The propagation of the diagnostic support for node $A_{8}$ from $A_{4}$ is obtained from the formula

$$
m_{A_{4} \uparrow A_{8}}=\alpha\left(m^{\prime}\left(A_{4}\right) \oplus m_{A_{7} \downarrow A_{4}} M_{A_{7} \downarrow A_{4}}^{T}\right)
$$

where $\alpha$ is a normalisation constant. On reference to the NTELECOM database in Appendix $\mathrm{A}$, the causal matrix for the link between nodes $A_{7}$ and $A_{4}$ is

$$
M_{A 7 \downarrow A_{4}}=\left[\begin{array}{lll}
289 / 317 & 28 / 317 & 0 \\
4 / 7 & 3 / 7 & 0 \\
0 & 0 & 1
\end{array}\right]=\left[\begin{array}{lll}
0.912 & 0.088 & 0 \\
0.517 & 0.427 & 0 \\
0 & 0 & 1
\end{array}\right]
$$

Therefore, $M_{A_{7} \downarrow A_{4}}^{T}=\left[\begin{array}{lll}0.615 & 0.385 & 0 \\ 0.170 & 0.830 & 0 \\ 0 & 0 & 1\end{array}\right]$ 


$$
\text { So, } \begin{aligned}
m_{A_{i} \uparrow \Lambda_{\mathrm{o}}} & =\alpha\left([0.62,0.25,0.13] \oplus[0,0,1]\left[\begin{array}{lll}
0.615 & 0.385 & 0 \\
0.17 .0 & 0.830 & 0 \\
0 & 0 & 0
\end{array}\right]\right) \\
& =[0.62,0.25,0.13]
\end{aligned}
$$

This updated bottom-up propagate mass function is now absorbed by the parent node $A_{8}$ for which the belief is updated. As shown in Fig. 13b, the evidence is now passed on to nodes $A_{1}$ and $A_{2}$ through top-down propagate mass functions $m_{A 8 \downarrow A 1}$ and $m_{A 3 \downarrow A 2}$, respectively. The calculations below show how $m_{A B \downarrow A 1}$ is updated.

\begin{tabular}{l|lll}
\multicolumn{4}{c}{$m_{A_{8} \downarrow A_{1}}^{\prime}=\alpha\left(m^{\prime}\left(A_{8}\right) \oplus m_{A_{4} \uparrow A_{8}}^{\prime}\right)$} \\
$m^{\prime}\left(A_{8}\right) \oplus m_{A_{4} A_{8}}^{\prime}$ & $\{0\}_{0.62}$ & $\{1\}_{0.25}$ & $\{0,1\}_{0.13}$ \\
\hline$\{0\}_{0.62}$ & $\{0\}_{0.384}$ & $\emptyset_{0.155}$ & $\{0\}_{0.081}$ \\
$\{1\}_{0.25}$ & $\emptyset_{0.155}$ & $\{1\}_{0.062}$ & $\{1\}_{0.033}$ \\
$\{0,1\}_{0.13}$ & $\{0\}_{0.081}$ & $\{1\}_{0.032}$ & $\{0,1\}_{0.017}$
\end{tabular}

\section{CONCLUSIONS}

After a presentation of a causal network generation, we used our novel genetic algorithm ( $C A E G A)$ to breed the most likely evidential causal network and showed how our Dempster-Shafer propagation scheme can be extended for a class of hierarchical networks capable of modeling multiple causes. Results from both a laboratory database and a real application in the telecommunications industry are presented. Belief parameters are updated by local computations, and once new evidence has passed through the network, the network reaches an equilibrium. The network-generation aspects of our reasoning algorithm (PropBel) were implemented and will be incorporated into a general-purpose knowledge discovery tool $M K S$, which provides linkage to databases and to libraries of discovery routines.

\section{ACKNOWLEDGMENT}

We would like to acknowledge the input of Mary Shapcott and the support of Northern Telecom in the development of this paper. 
APPENDIX A:

TABLE 4

Database (NTELECOM) Sample Storing 8 Binary Variables

\begin{tabular}{|c|c|c|c|c|c|c|c|c|}
\hline$A_{1}$ & $A_{2}$ & $A_{3}$ & $A_{4}$ & $A_{5}$ & $A_{6}$ & $A_{7}$ & $A_{\mathbf{B}}$ & Freq. \\
\hline 1 & 0 & 0 & 0 & 0 & 0 & 0 & 0 & 13 \\
\hline 0 & 1 & 0 & 0 & 0 & 0 & 0 & 0 & 68 \\
\hline 1 & 1 & 0 & 0 & 0 & 0 & 0 & 0 & 8 \\
\hline 0 & 0 & 1 & 0 & 0 & 0 & 0 & 0 & 11 \\
\hline 1 & 0 & 1 & 0 & 0 & 0 & 0 & 0 & 2 \\
\hline 0 & 1 & 1 & 0 & 0 & 0 & 0 & 0 & 46 \\
\hline 1 & 1 & 1 & 0 & 0 & 0 & 0 & 0 & 6 \\
\hline 0 & 0 & 0 & 1 & 0 & 0 & 0 & 0 & 4 \\
\hline 1 & 0 & 0 & 1 & 0 & 0 & 0 & 0 & 1 \\
\hline 1 & 1 & 0 & 1 & 0 & 0 & 0 & 0 & 2 \\
\hline 0 & 0 & 1 & 1 & 0 & 0 & 0 & 0 & 2 \\
\hline 1 & 0 & 1 & 1 & 0 & 0 & 0 & 0 & 1 \\
\hline 0 & 1 & 1 & 1 & 0 & 0 & 0 & 0 & 2 \\
\hline 1 & 1 & 1 & 1 & 0 & 0 & 0 & 0 & 2 \\
\hline 0 & 0 & 0 & 0 & 1 & 0 & 0 & 0 & 77 \\
\hline 1 & 0 & 0 & 0 & 1 & 0 & 0 & 0 & 4 \\
\hline 0 & 1 & 0 & 0 & 1 & 0 & 0 & 0 & 2 \\
\hline 0 & 0 & 1 & 0 & 1 & 0 & 0 & 0 & 2 \\
\hline 1 & 0 & 1 & 0 & 1 & 0 & 0 & 0 & 3 \\
\hline 0 & 1 & 1 & 0 & 1 & 0 & 0 & 0 & 2 \\
\hline 1 & 1 & 1 & 0 & 1 & 0 & 0 & 0 & 4 \\
\hline 0 & 0 & 1 & 1 & 1 & 0 & 0 & 0 & 1 \\
\hline 1 & 0 & 1 & 1 & 1 & 0 & 0 & 0 & 2 \\
\hline 0 & 1 & 1 & 1 & 1 & 0 & 0 & 0 & 2 \\
\hline 1 & 1 & 1 & 1 & 1 & 0 & 0 & 0 & 1 \\
\hline 0 & 0 & 0 & 0 & 0 & 1 & 0 & 0 & 23 \\
\hline 1 & 0 & 0 & 0 & 0 & 1 & 0 & 0 & 3 \\
\hline 0 & 1 & 0 & 0 & 0 & 1 & 0 & 0 & 1 \\
\hline 1 & 1 & 0 & 0 & 0 & 1 & 0 & 0 & 2 \\
\hline 1 & 0 & 1 & 0 & 0 & 1 & 0 & 0 & 2 \\
\hline 1 & 1 & 1 & 0 & 0 & 1 & 0 & 0 & 1 \\
\hline 0 & 0 & 0 & 1 & 0 & 1 & 0 & 0 & 1 \\
\hline 1 & 1 & 0 & 1 & 0 & 1 & 0 & 0 & 4 \\
\hline 1 & 1 & 1 & 1 & 0 & 1 & 0 & 0 & 2 \\
\hline 0 & 0 & 0 & 0 & 1 & 1 & 0 & 0 & 7 \\
\hline 0 & 0 & 0 & 0 & 0 & 0 & 1 & 0 & 2 \\
\hline 1 & 1 & 0 & 0 & 0 & 0 & 1 & 0 & 1 \\
\hline 0 & 0 & 0 & 1 & 0 & 0 & 1 & 0 & 1 \\
\hline 1 & 0 & 1 & 1 & 1 & 0 & 1 & 0 & 1 \\
\hline 1 & 1 & 1 & 0 & 0 & 1 & 1 & 0 & 1 \\
\hline 1 & 1 & 1 & 1 & 0 & 1 & 1 & 0 & 1 \\
\hline 1 & 1 & 1 & 0 & 0 & 0 & 0 & 0 & 1 \\
\hline 0 & 0 & 0 & 1 & 0 & 0 & 0 & 0 & 1 \\
\hline & 0 & 0 & 0 & 0 & 1 & 0 & 0 & 1 \\
\hline & & & & & & & & 324 \\
\hline 1 & & & & & & \\
\hline
\end{tabular}




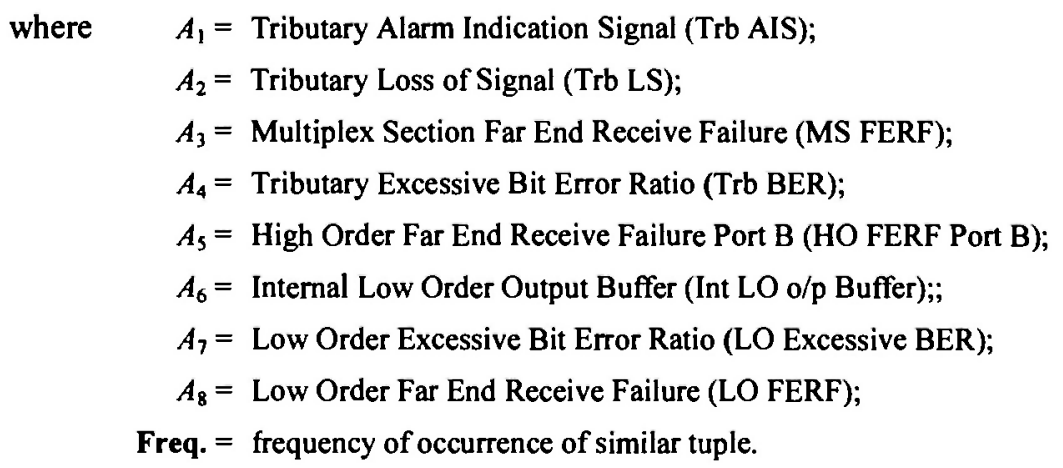

\section{APPENDIX B:}

\section{The Concept of Causation}

Many philosophers (such as Hume and Kant) have asserted that causation cannot be validated by direct observation, and the concept of causation is not amenable to validation by logic construction because it is an empirical concept. Yet causal connection is seen to be fundamentally required if we are to conceive of an ordered world. Working from sense data alone is insufficient to capture the notion completely. There is no third entity between events $C$ and $\varepsilon$, (a cause and an effect, respectively) that we can observe. While acknowledging this fact, we wish to see how far we can get from sense data alone, in relatively simple physical (not cognitive, social, or even biological) systems, and our hypothesis is that we can get quite far (McKearney et al., 1992). The output will always have to be supplemented by exogenous knowledge, of course.

Our working conjecture inspiring this work is that, as is the case in the 'constructive' approach to the stating of personal preferences and the allocating of probabilities (Shafer \& Srivastava, 1990), for example, people do not generally know causes and effects. When asked what the cause of something is, they look for arguments on which to base their choices (cf. Bell, 1993). Our idea is that canonical examples of the concept of cause can be of great help here. People can then say by cause I mean an "analogy with... relationship".

Without such canonical examples, the arguments for causality can be very inconsistent. In the assessment of preferences, for example, there are all 
sorts of ways in which the alternatives are presented to the choice-maker, and these suggest different arguments, and therefore influence the choices made. This can, of course, lead to inconsistency: "inescapable for rational beings who base their choices on arguments" (Shafer, 1986).

Such inconsistency is highly undesirable when predications on the question of mechanical, safety-critical systems, such as cars, planes, and space shuttles, are the final goals. In such physical worlds, a tighter coupling, and hence predictability, of arguments' relationships to choices is a necessity. Clearly, formulae are very useful devices in such circumstances, but if formulae are not available, a sort of 'half-way house' is required. In this compromise, the extremes of taking the very subjective view on the one hand and requiring a 'completely specified' view on the other hand are avoided.

As indicated above, an approach to this problem is suggested in the case of subjective probability assessments (Shafer \& Srivastava, 1990). Arguments here, according to the constructive interpretation, use probability formulations that are based on "assessing the strength and structure of evidence by comparing to a scale of canonical examples". In the general assessment of preferences, however, things are somewhat more loosely determined.

We have suggested elsewhere that this sort of half-way-house approach is suitable for an understanding of the concept of cause (Bell \& McErlean, 1995), which is useful in cases where "simple physical systems" only are involved. A working understanding of the word 'cause', for use in KDD among other activities, can be based on these simple 'canonical' causal relations.

Causes and effects are not quite to difficult to pin down in an 'ideal physical world' (see below). This 'world' can give insights into real-world behavior.

Our viewpoint is that "by paralleling well-understood (canonical) ideal physical worlds in this way we obtain a world model which is useful". Being useful is, like the quality of being 'interesting' in common KDD parlance, an application-sensitive quality. We assume, however, without further discussion that the word has an intuitive notion associated with it, which is so widely accepted that it allows us to apply the word to things and actions in an acceptably meaningful manner.

So our concept of cause and effect is one based on a canonical metaphor. We adopt a simple, conceptual definition of cause and effect in terms of 
canonical, relatively unambiguous, ('causally') related events, which we label $C$ and $\varepsilon$, respectively. We use this by applying the following template:

$$
\text { " } A \text { " is to " } B \text { " as " } C \text { " is to " } \mathrm{".}
$$

" $A$ " and " $B$ " here are two events of interest. If they together parallel the $C$ to $\varepsilon$ relationship, we can say

$$
A \text { "causes" } B
$$

in the same manner as

$$
\text { C"causes" } \varepsilon .
$$

Now what canonical exemplar of the concept do we use? We assume for the purposes of this paper that the causal relationship of C "causes" $\varepsilon$ is a rich amalgam ("an amicably confused jumble") of the notions (Skyrms, 1984).

" $\varepsilon$ is statistically relevant to $C$ "

"changing parts of $C$ will cause corresponding parts of $\varepsilon$ to change"

"there is some proximity/succession relationship between $\varepsilon$ and $C$ "

' $C$ explains $\varepsilon$ '.

The jumble should also include the condition

"A cause is a ceteris paribus sufficient and necessary condition for the effects."

- so long as we take a probabilistic interpretation of necessity and sufficiency (Scriven, 1975).

The canonical scenario, which we use to capture the concept of cause for the purposes of this paper, is presented in formally as a simple ithought experimentj as follows:

Consider a plane inclined at about $15^{\circ}$ to the horizontal. It has a hole in it, with a radius identically equal to that of a ball; the plane covers a space below and light can enter the space through the hole. The ball is rolled down the plane, along a guide that delivers it to the hole. The ball will stop, in an ideal physical world and the space under the hole will no longer be illuminated.

(Think of a table-tennis ball rolling down the box-lid, which has a hole in it.)

The $C$ for this canonical case is the ball rolling, and the $\varepsilon$ is the light being put out. We have argued elsewhere (Bell \& McErlean, 1995) that his 
scenario meets the requirements for cause and effect above. The thought experiment has been used as the basis of a physical experiment, where because the plane was not perfectly smooth: (it was, in fact, a box-lid!), and so on, sometimes the (table-tennis) ball does not cover the hole, but bounces over it. So in actual physical worlds, the relation of $C$ to $\varepsilon$ is probablistic.

This simple physical $C-\varepsilon$ relationship avoids difficulties that are raised when cognitive agents, etc., are involved. For example, a faction tries to overthrow a government, and the army waits in the wings to see if the faction is successful, only intervening if it is unsuccessful. (This sort of contingency can be modeled to some extent in our simple physical world by a golf ball following the table-tennis ball, and only (probably) causing the darkening effect if the table-tennis ball does not do so, but there is an argument saying that this model is incomplete.) As an example of the application of this canonical approach to definition, in our network in Fig. 1, most of the edges of the graph can be interpreted in an acceptable manner according to the template above, using the given Cause-Effect pairing.

\section{REFERENCES}

Agrawal, R., Imielinski, T., Swami, A. 1993. Database mining : A performance perspective. IEEE Transactions on Knowledge and Data Engineering. Special Issue on Learning and Discovery in Knowledge-base Databases, 5, 914-925.

Anand, S., Bell, D., and Hughes, J. 1996. A general framework for data mining based on evidence theory. Data and Knowledge Engineering, 18, 189-223.

Bell, D. 1993. From data properties to evidence. IEEE Transactions on Knowledge and Data Engineering. Special Issue on Learning and Discovery in Knowledge-based Databases, 56, 965-968.

Bell, D. and McErlean, F. 1995. A multi-source approach to the generation of cause-and- effect networks from datatabases. Technical report, University of Ulster, Shore Road, Jordanstown, North Ireland. 
Bell, D., Guan, J., and Lee, S. 1996. Generalised union and projection operators for pooling uncertain and imprecise information. Data and Knowledge Engineering, 18, 89-117.

Cao, H., Bell, D., and Hull, M. 1993. Multi-level parallelism for evaluation of deductive database queries, in: Proceedings of the International Conference YCS, 544-547.

Cheng, J., Bell, D., and Liu, W. 1997. An algorithm for Bayesian belief network construction from data. In: Proceedings of the Sixth International Workshop on Artificial Intelligence and Statistics.

Chow, C. and Liu, C. 1968. Approximating discrete probability distribution with dependence trees. IEEE Transaction on Information Theory, IT-143, 462-467.

Cooper, G. and Herskovits, E. 1992. A Bayesian method for the induction of probabilistic networks from data. machine learning, 9, 309-347.

Druzdzel, M. and Simon, H. 1993. Causality in Bayesian belief networks, in: Proceedings of Uncertainty in Artificial Intelligence, 3-11.

Dubois, D. and Prade, H. 1990. An introduction to possibilistic and fuzzy logics, in: Readings in uncertain reasoning, edited by Shafer, G. and Pearl, J., San Mateo, California, USA, Morgan Kaufmann, 742-761.

Goldberg, D. 1989. Genetic algorithms in search, optimisation, and learning. New York, NY, USA, Addison-Wesley.

Gordon, J., and Shortliffe, E. 1984. The Dempster-Shafer theory of evidence, in: Rule-based expert systems: The MYCIN experiments of the Stanford Heuristic Programming project, Chapter 13. Reading, Massachussets, USA, Addison- Wesley.

Guan, J., and Bell, D. 1991. Evidence theory and its applications, Volume 1. Amsterdam, The Netherlands, North-Holland.

Larranaga, P. 1995. Genetic algorithms applied to Bayesian networks, in: Proceedings of Applied Decision Technologies.

Magill, I., Bell, D.A., Hughes, J.G., and Anand, S., Tan, M. 1995. Database mining in the Northern Ireland housing executive, in: Proceedings of the IEE Colloquium on Knowledge Discovery in Databases, 81-84.

McErlean, F., Bell, D., and Guan J. (in press). Modification of belief in evidential causal networks. IST Journal. 
McKearney, S., Bell, D., and Hickey, R. 1992. Inferring abstract objects in databases, in: Proceedings of the International Conference of CIKM.

Neapolitan, R. 1990. Probabilistic Reasoning in Expert Systems: Theory and Algorithms. New York, NY, USA, J. Wiley and Sons.

Pawlak, Z. 1982. Rough Sets, International Journal of Computer and Information Sciences, 11, 341-356.

Pawlak, Z. 1991. Rough sets: Theoretical aspects of reasoning about data. Dordrecht, The Netherlands, Kluwer Academic Publishers.

Pearl, J. 1986. Fusion, propagation, and structuring in belief networks. Artificial Intelligence, 37, 241-288.

Pearl, J. 1988. Probabilistic reasoning in intelligent systems: Networks of plausible inference, 2nd edition. San Francisco, CA, USA, Morgan Kaufmann Publishers, Inc.

Rojas-Guzman, C., and Kramer, M. 1993. GALGO: A Genetic ALGOrithm decision support tool for complex uncertain systems modeled with Bayesian belief networks, in: Proceedings of Uncertainty in Artificial Intelligence.

Scriven, M. 1975 Defects of the necessary condition analysis of causation and conditioning. Oxford, UK, Oxford University Press.

Shafer, G. 1976. A mathematical theory of evidence. Princeton, NJ, USA, Princeton University Press.

Shafer, G. 1986. Savage revisited. Statistical Science, 1, 463-485.

Shafer, G., and Srivastava, R. 1990. The Bayesian and belief-function formalism, in: Uncertain reasoning, edited by Shafer G., and Pearl, J., San Mateo, California, USA, Morgan Kaufmann.

Shannon, C., and Weaver, W. 1949. The Mathematical Theory of Communication. Urbana, IL, USA, University of Illinois Press.

Simon, H. 1953. Causal ordering and identifiability, in: Studies in econometric method, Chapter 3, edited by Hood W., and Hoopmans, T. New York, NY, USA, J. Wiley and Sons, 49-74.

Skowran, A., and Grzymala-Busse, J. 1994. From rough set theory to evidence theory, in: Advances in the Dempster-Shafer theory of evidence, edited by Yaeger, R., Kacprzyk, J., and Fedrizzi, M., New York, NY, USA, J. Wiley and Sons. 
Skyrms, B. 1984. EPR: Lessons for metaphysics, in: Midwest studies in philosophy, Vol. 1, Causation and Causal Theories, edited by French, P., T.U. Jr, and Wettstein, H., Minneapolis, MN, USA University of Minnesota Press.

Sterritt, R. Adamson, K., Shapcott, M. Bell, D.A., and McErlean, F. 1997a. NETEXTRACT: An architecture for the extraction of cause and effect networks from complex systems, in: Proceedings of the 15th IASTED International Conference on APPLIED INFORMATICS, 55-57.

Sterritt, R. Adamson, K., Shapcott, M. Bell, D.A., and McErlean, F. 1997b. Using A.I. for the Analysis of Complex Systems, in: Proceedings of the IASTED International Conference on ARTIFICIAL INTELLIGENCE AND SOFT.COMPUTING, 113-116.

Whitley, D. 1987. Using reproductive evaluation to improve genetic search and heuristic discovery, in: Proceedings of the Second International Conference on Genetic Algorithms, 108-115. 